\title{
SOME NEW CONSTRUCTIONS AND ESTIMATES IN THE PROBLEM OF LEAST AREA ${ }^{1}$ \\ BY
}

\author{
HAROLD PARKS
}

\begin{abstract}
Surfaces of least $k$ dimensional area in $\mathbf{R}^{n}$ are constructed by minimization of the $\boldsymbol{n}$ dimensional volume of suitably thickened sets subject to a homological constraint. Specifically, let $1<k<n$ be integers and $B \subset \mathbf{R}^{n}$ be compact and $k-1$ rectifiable. Let $G$ be a compact abelian group and $L$ be a subgroup of the Cech homology group $H_{k-1}(B ; G)$ (in case $k=1$, suppose, additionally, $L$ is contained in the kernel of the usual augmentation map). J. F. Adams has defined what it means for a compact set $X \subset \mathbf{R}^{n}$ to span $L$. Using also a natural notion of what it means for a compact set to be $\varepsilon$-thick, we show that, for each $\varepsilon>0$, there exists an $\varepsilon$-thick set which minimizes $n$ dimensional volume subject to the requirement that it span $L$. Our main result is that as $\varepsilon$ approaches 0 a subsequence of the above volume minimizing sets converges in the Hausdorff distance topology to a set, $X$, which minimizes $k$ dimensional area subject to the requirement that it span $L$. It follows, of course, from the regularity results of Reifenberg or Almgren that, except for a compact singular set of zero $k$ dimensional measure, $X$ is a real analytic minimal submanifold of $\mathbf{R}^{n}$.
\end{abstract}

1. Introduction. Consider a compact $k-1$ rectifiable set $B \subset \mathbf{R}^{n}(1<k \leqslant$ $n$ ), a compact abelian group $G$, and a subgroup $L$ of the Čech homology group $H_{k-1}(B ; G)$ (in case $k=1$, one supposes, additionally, $L$ is contained in the kernel of the usual augmentation map). A compact set $X \subset \mathbf{R}^{n}$ is said to span $L$ if and only if $B \subset X$ and $i_{*}(L)=0$ where $i: B \rightarrow X$ is the inclusion map. Typically one seeks a compact set $X \subset \mathbf{R}^{n}$ spanning $L$ such that

$$
\mathcal{H}^{k}(X)=\inf \left\{\mathcal{H}^{k}(Y): Y \subset \mathbf{R}^{n} \text { is compact and spans } L\right\}
$$

on the other hand, it is usually considerably easier to find a compact set $X \subset \mathbf{R}^{n}$ spanning $L$ such that

$$
\begin{array}{r}
\mathcal{L}^{n}\{x: \operatorname{dist}(x, X) \leqslant \varepsilon\}=\inf \left\{\mathcal{L}^{n}\{y: \operatorname{dist}(y, Y) \leqslant \varepsilon\}:\right. \\
\left.Y \subset \mathbf{R}^{n} \text { is compact and spans } L\right\} ;
\end{array}
$$

here $\varepsilon>0$ and $\mathcal{F}^{k}$ and $\mathfrak{L}^{n}$ denote Hausdorff $k$ dimensional measure on $\mathbf{R}^{n}$ and Lebesgue $n$ dimensional measure, respectively. The following theorem

Received by the editors May 13, 1977.

AMS (MOS) subject classifications (1970). Primary 49F22, 49D30.

Key words and phrases. Homological constraint, Hausdorff measure, Hausdorff distance, polyhedral complexes.

'This paper was prepared while the author was at Brown University. 
relates these two procedures. Let $\varepsilon_{1}, \varepsilon_{2}, \varepsilon_{3}, \ldots>0$ with $\lim _{i} \varepsilon_{i}=0$.

TheOREM (1) For each $i=1,2,3, \ldots$ there exists a compact set $X_{i} \subset \mathbf{R}^{n}$ which spans $L$ and satisfies (*) with $\varepsilon$ replaced by $\varepsilon_{i}$.

(2) For $X_{1}, X_{2}, X_{3}, \ldots$ chosen as in (1) there exists a subsequence $i(1), i(2)$, $i(3), \ldots$ of $1,2,3, \ldots$ and a compact set $X \subset \mathbf{R}^{n}$ such that $\lim _{j} X_{i(j)}=X$ in the Hausdorff distance topology, $X$ spans $L$, and

$$
\mathcal{H}^{k}(X)=\inf \left\{\mathcal{H}^{k}(Y): Y \subset \mathbf{R}^{n} \text { is compact and spans } L\right\}
$$

It then follows from the regularity results of Reifenberg or Almgren that, except for a compact singular set of zero $\mathcal{H}^{k}$ measure, $X$ is a real analytic minimal submanifold of $\mathbf{R}^{n}$.

The central tool used in proving the above theorem is a correspondence between $\varepsilon$-thick sets and polyhedral complexes (as defined in \$5.1) based on Besicovitch's covering theorem. Polyhedral complexes, in particular, admit useful estimates on $\mathfrak{L}^{n}\{x: \operatorname{dist}(x, \cdot) \leqslant \varepsilon\}$ and also admit various intricate geometric constructions (see, for example, the Isoperimetric Theorem 5.3).

2. Definitions. Except when otherwise stated, we will follow the notation and terminology of [FH]. Denote by $n$ and $k$ integers with $1<k<n$ and by $G$ a compact, abelian group.

(1) Set $\mathbf{R}^{+}=\mathbf{R} \cap\{t: t>0\}$.

(2) For each positive integer $m$, each $a \in \mathbf{R}^{m}$, and each $r \in \mathbf{R}^{+}$define the closed [respectively, open] ball of radius $r$ centered at $a, \mathbf{B}^{m}(a, r)$ [respectively, $\mathbf{U}^{m}(a, r)$, by setting

[respectively,

$$
\mathbf{B}^{m}(a, r)=\mathbf{R}^{m} \cap\{x:|x-a| \leqslant r\}
$$

$$
\left.\mathbf{U}^{m}(a, r)=\mathbf{R}^{m} \cap\{x:|x-a|<r\}\right]
$$

and the sphere of radius $r$ centered at $a, S^{m-1}(a, r)$, by setting

$$
\mathbf{S}^{m-1}(a, r)=\mathbf{R}^{m} \cap\{x:|x-a|=r\}
$$

also, set

$$
\mathrm{Ct}\left[\mathbf{B}^{m}(a, r)\right]=\mathrm{Ct}\left[\mathbf{U}^{m}(a, r)\right]=a,
$$

and for each $A \subset \mathbf{R}^{m}$ and each (closed or open) ball $B \subset \mathbf{R}^{m}$, say $B$ is centered in $A$ if and only if $\operatorname{Ct}(B) \in A$.

(3) Set

$$
\bigodot_{0}=\left\{C: C \text { is a compact subset of } \mathbf{R}^{n}\right\} \text { and } e=\bigodot_{0} \sim\{\varnothing\} .
$$

(4) Define the Hausdorff distance $d: \mathcal{C} \times \mathcal{C} \rightarrow \mathbf{R}$ by setting

$$
\begin{aligned}
d\left(C_{1}, C_{2}\right)=\sup \left\{\operatorname{dist}\left(x, C_{i}\right): i=1\right. & \text { and } x \in C_{2}, \\
& \text { or } \left.i=2 \text { and } x \in C_{1}\right\}
\end{aligned}
$$

for each $\left(C_{1}, C_{2}\right) \in \mathcal{C} \times \mathcal{C}$. 
(5) For each $\varepsilon \in \mathbf{R}^{+}$, define $c_{\varepsilon}, e_{\varepsilon}: \bigodot_{0} \rightarrow \bigodot_{0}$ by setting

$$
c_{\varepsilon}(C)=\mathbf{R}^{n} \cap\left\{x: \operatorname{dist}\left(x, \mathbf{R}^{n} \sim C\right) \geqslant \varepsilon\right\} \subset C
$$

and

$$
e_{\varepsilon}(C)=\mathbf{R}^{n} \cap\{x: \operatorname{dist}(x, C) \leqslant \varepsilon\} \supset C
$$

for each $C \in \mathcal{C}_{0}$.

(6) For each $\varepsilon \in \mathbf{R}^{+}$, set

$$
\bigodot_{\varepsilon}=\bigodot \cap\left\{C: e_{\varepsilon}: c_{\varepsilon}(C)^{\circ}=C\right\}
$$

the elements of $C_{e}$ are called $\varepsilon$-thick sets.

(7) For each positive integer $m$, each $A \subset \mathbf{R}^{m}$, and each $B \subset \mathbf{R}^{m}$ set

$$
A * B=\{t a+(1-t) b: a \in A, b \in B, 0<t<1\}
$$

and set $\operatorname{Cv}(A)$ equal to the convex hull of $A$.

(8) For each positive integer $m$ and each sequence $x_{0}, x_{1}, x_{2}, \ldots, x_{m} \in \mathbf{R}^{n}$ set

$$
\begin{aligned}
\left\langle x_{0}, x_{1}, x_{2}, \ldots, x_{m}\right\rangle & \\
& =\left\{\sum_{i} \lambda_{i} x_{i}: 0<\lambda_{i} \text { for } i=0,1,2, \ldots, m \text { and } \sum_{i} \lambda_{i}=1\right\}
\end{aligned}
$$

and set $\left\langle x_{0}\right\rangle=\left\{x_{0}\right\}$.

(9) For each $A \subset \mathbf{R}^{n}$ and each

$$
\mathbb{Q} \subset\left\{\mathbf{U}^{n}(x, r): x \in \mathbf{R}^{n}, r \in \mathbf{R}^{+}\right\}
$$

with $A \subset \cup \mathbb{Q}$, set

$$
\begin{aligned}
& \mathbf{N}(\mathscr{Q}, A)=\bigcup_{m>0} \cup\left\{\left\langle\operatorname{Ct}\left(U_{0}\right), \operatorname{Ct}\left(U_{1}\right), \operatorname{Ct}\left(U_{2}\right), \ldots, \operatorname{Ct}\left(U_{m}\right)\right\rangle:\right. \\
&\left.U_{i} \in \mathscr{Q} \text { for } i=0,1,2, \ldots, m \text { and } A \cap \bigcap_{i} U_{i} \neq \varnothing\right\} .
\end{aligned}
$$

(10) For each $x=\left(x_{1}, x_{2}, x_{3}, \ldots, x_{n}\right) \in \mathbf{R}^{n}$ set

$$
\mathbf{m}(x)=\sup \left\{\left|x_{i}\right|: i=1,2,3, \ldots, n\right\} .
$$

(11) Whenever $X, A \in \mathcal{C}_{0}$ with $A \subset X$ and $q$ is a nonnegative integer, we denote by $H_{q}(X, A)$ the $q$ dimensional Čech homology group of $X$ relative to $A$ with coefficients in $G$ (see [ES, IX]). Each continuous mapping

$$
f:(X, A) \rightarrow(Y, B),
$$

$Y, B \in \mathcal{C}_{0}, B \subset Y$, induces a homomorphism

$$
f_{*}: H_{q}(X, A) \rightarrow H_{q}(Y, B)
$$

in the usual way. Set $H_{q}(X)=H_{q}(X, \varnothing)$ and let

$$
\operatorname{aug}(X): H_{0}(X) \rightarrow G
$$

be the augmentation map induced by the map from $X$ to the one point space. 
(12) For each $B \in \mathcal{C}$ and each subgroup $L \subset H_{k-1}(B)$ we say that $X \in \mathcal{C}$ spans $L$ if and only if $B \subset X$ and $i_{*}(L)=0$ where $i: B \rightarrow X$ is the inclusion map. Denote by $\mathcal{C}(B, L)$ the collection of sets in $\mathcal{C}$ which span $L$ and, for each $\varepsilon \in \mathbf{R}^{+}$, set

$$
\begin{gathered}
\mu_{\varepsilon}(B, L)=\inf \left\{\mathfrak{L}^{n} \circ e_{\varepsilon}(X): X \in \mathcal{C}(B, L)\right\}, \\
\mathfrak{T}_{\varepsilon}(B, L)=\left\{X \in \mathcal{C}(B, L): \mathscr{L}^{n} \circ e_{\varepsilon}(X)=\mu_{\varepsilon}(B, L)\right\} .
\end{gathered}
$$

\section{An existence theorem.}

TheOREM. (1) For each $B \in \mathcal{C}$ and each subgroup $L$ of $H_{k-1}(B)$, which in case $k=1$ satisfies $L \subset \operatorname{ker}[\operatorname{aug}(B)], \mathcal{C}(B, L)$ is nonempty.

(2) For each $r \in \mathbf{R}^{+}, \mathcal{C} \cap\left\{C: C \subset \mathbf{B}^{n}(0, r)\right\}$ is compact in the Hausdorff distance topology.

(3) For each $\varepsilon \in \mathbf{R}^{+}, e_{\varepsilon} \mid \mathcal{C}: \mathcal{C} \rightarrow \bigodot_{\varepsilon}$ is continuous in the Hausdorff distance topology.

(4) For each $\varepsilon \in \mathbf{R}^{+}, \mathfrak{L}^{n}: \bigodot_{\varepsilon} \rightarrow \mathbf{R}^{+}$is continuous in the Hausdorff distance topology.

(5) For each $B \in \mathcal{C}$ and each subgroup $L$ of $H_{k-1}(B), \mathcal{C}(B, L)$ is a closed subset of $\mathcal{C}$ in the Hausdorff distance topology.

(6) For each $\varepsilon \in \mathbf{R}^{+}$, each $B \in C$, and each subgroup $L$ of $H_{k-1}(B)$, which in case $k=1$ satisfies $L \subset \operatorname{ker}[\operatorname{aug}(B)], \mathfrak{T}_{e}(B, L)$ is nonempty.

Proof. (1) Conclusion (1) follows from [RE, Lemma 2A] applied to $\{0\} * B$.

(2) Conclusion (2) is contained in [FH, 2.10.21].

(3) First, we check that $e_{\varepsilon}(\mathcal{C}) \subset \mathcal{C}_{\varepsilon}$. Let $C \in \mathcal{C}$ be arbitrary. We must show that

$$
e_{\varepsilon} \circ c_{\varepsilon}\left[e_{\varepsilon}(C)\right]=e_{\varepsilon}(C) .
$$

If $x \in e_{\varepsilon} \circ c_{\varepsilon}\left[e_{\varepsilon}(C)\right]$, then there is $y \in c_{\varepsilon}\left[e_{\varepsilon}(C)\right]$ with $|x-y|<\varepsilon$, but, since $y \in c_{e}\left[e_{\varepsilon}(C)\right]$, we have $\mathbf{B}^{n}(y, \varepsilon) \subset e_{\varepsilon}(C)$, so $x \in e_{\varepsilon}(C)$; on the other hand, if $x \in e_{\varepsilon}(C)$, then there is $y \in C$ with $|x-y| \leqslant \varepsilon$, so $\mathbf{B}^{n}(y, \varepsilon) \subset e_{\varepsilon}(C)$ and thus $y \in c_{\varepsilon}\left[e_{\varepsilon}(C)\right]$, so $x \in e_{\varepsilon} \circ c_{\varepsilon}\left[e_{\varepsilon}(C)\right]$.

Next, we check the continuity of $e_{\varepsilon} \mid \mathcal{C}$. Assume $C, D \in \mathcal{C}$ and $b \in e_{\varepsilon}(C)$. Then there exist $c \in C$ and $d \in D$ such that $|b-c|<\varepsilon$ and $|c-d|<$ $d(C, D)$. In the line segment $b d$ one can clearly choose $e \in e_{e}(D)$ with $|b-e| \leqslant d(C, D)$. Similarly, for each $e^{\prime} \in e_{\varepsilon}(D)$ there exists $b^{\prime} \in e_{\varepsilon}(C)$ with $\left|b^{\prime}-e^{\prime}\right| \leqslant d(C, D)$. Thus we have

$$
d\left[e_{\varepsilon}(C), e_{\varepsilon}(D)\right] \leqslant d(C, D) .
$$

(4) Assume $C \in \mathcal{C}_{\varepsilon}$ and let $0<\lambda<1$ be arbitrary. Set $\tau_{1}=\lambda^{1 / 3}$ and $\tau_{2}=2^{-1} \tau_{1}$. Choose an integer $m$ such that $\sum_{i=0}^{m}\left(\tau_{1} / 2\right)^{i}>2 \lambda^{1 / 3}, \tau_{3}$ such that 
$\tau_{2}<\tau_{3}<2^{-1}$, and $\sigma_{0} \in \mathbf{R}^{+}$such that for each $(p, q) \in \mathbf{R}^{n} \times \mathbf{R}^{n}$, with $\mid p-$ $q \mid=\varepsilon$, and each $0<\sigma<\sigma_{0}$ one has

$$
\mathcal{L}^{n}\left[\mathbf{B}^{n}(p, \sigma) \cap \mathbf{B}^{n}(q, \varepsilon)\right] \geqslant \tau_{3} \boldsymbol{\alpha}(n) \sigma^{n} .
$$

Set

$$
U^{+}=\mathbf{U}^{n}(0,1) \cap\left\{x: x \cdot \mathbf{e}_{n}>0\right\}
$$

and

$$
U^{-}=\mathbf{U}^{n}(0,1) \cap\left\{x: x \cdot \mathbf{e}_{n}<0\right\} .
$$

By [FH, 2.8.15] there exists a finite disjointed family $F$ of closed balls contained in $U^{+}$such that

$$
\mathcal{L}^{n}(\cup F) \geqslant \tau_{1} \mathcal{L}^{n}\left(U^{+}\right)
$$

Set $\alpha=\inf \left\{2^{-1} \operatorname{diam} S: S \in F\right\}$; note that $\alpha<2^{-1}$.

Since $C=e_{\varepsilon} \circ c_{\varepsilon}(C)$, by [FH, 3.2.34], one has

$$
\begin{aligned}
\mathfrak{L}^{n}(C) & \leqslant \mathscr{L}^{n}\left[c_{\varepsilon}(C)\right]+\lim _{t \rightarrow \varepsilon+} \int_{0}^{t} \mathcal{H}^{n-1}\left\{x: \operatorname{dist}\left[x, c_{\varepsilon}(C)\right]=s\right\} d \mathfrak{L}_{s}^{1} \\
& =\mathfrak{L}^{n}\left[c_{\varepsilon}(C)\right]+\int_{0}^{\varepsilon} \mathcal{F}^{n-1}\left\{x: \operatorname{dist}\left[x, c_{\varepsilon}(C)\right]=s\right\} d \mathfrak{L}_{s}^{1} \\
& =\mathfrak{L}^{n}(\operatorname{Int} C) \leqslant \mathfrak{L}^{n}(C) .
\end{aligned}
$$

Thus $\mathfrak{L}^{n}(C)=\mathfrak{L}^{n}($ Int $C)$ and, applying [FH, 2.8.15], one obtains a finite disjointed family, $G_{0}$, of closed balls contained in Int $C$ of radius less than $\sigma_{0}$ such that

$$
\mathcal{L}^{n}\left(\cup G_{0}\right) \geqslant \tau_{1} \varrho^{n}(C) .
$$

Set $\beta_{0}=\inf \left\{2^{-1} \operatorname{diam} S: S \in G_{0}\right\}$. Choose $\delta_{0} \in \mathbf{R}^{+}$such that for each $p \in$ $\mathbf{R}^{n}$, each $q \in \mathbf{R}^{n}$, each $\sigma$ with $\alpha^{m} \beta_{0} \leqslant \sigma<\sigma_{0}$, and each $\delta$ with $0<\delta<\delta_{0}$ : If $|p-q|=\varepsilon+\delta$, then

$$
\mathcal{L}^{n}\left[\mathbf{B}^{n}(p, \sigma) \cap \mathbf{B}^{n}(q, \varepsilon)\right] \geqslant \tau_{2} \boldsymbol{\alpha}(n) \boldsymbol{\sigma}^{n} .
$$

Now, fix $D \in \mathcal{C}_{e}$ with $d(C, D)<\delta_{0}$. We will construct inductively certain families, $G_{i}$, of closed balls contained in Int $C$ and certain Borel subsets, $E(i)$, of $D \cap$ Int $C$, for $0 \leqslant i \leqslant m+1$, such that

(a) $G_{i}$ is disjointed;

(b) if $S \in G_{i}$ and $r=2^{-1} \operatorname{diam} S$, then $\alpha^{i} \beta_{0} \leqslant r<\sigma_{0}$;

(c) if $1<i \leqslant m+1$, then

$$
\cup G_{i} \subset \cup G_{i-1} \text { and } \mathscr{L}^{n}\left(\cup G_{i}\right)>\tau_{2} \mathscr{L}^{n}\left(\cup G_{i-1}\right) \text {; }
$$

(d) $E(0)=\varnothing$ and if $1 \leqslant i \leqslant m+1$, then

$$
E(i) \subset \cup G_{i-1} \sim \cup G_{i} \text { and } \mathfrak{L}^{n}(E(i))>\tau_{2} \mathscr{L}^{n}\left(\cup G_{i-1}\right) .
$$

Suppose $G_{i}$ and $E(i)$ have been constructed and $0<i<m$. For each $S \in G_{i}$, because $\operatorname{Ct}(S) \in C, D \in \mathcal{C}_{e}, d(C, D)<\delta_{0}$, and 


$$
\alpha^{m} \beta_{0} \leqslant 2^{-1} \operatorname{diam} S<\sigma_{0},
$$

there exists an isometry $h_{S}$ of $\mathbf{R}^{n}$ so that

$$
h_{S} \circ \mu_{r}\left[\mathbf{B}^{n}(0,1)\right]=S \text { and } \mathcal{L}^{n}\left(E_{S}\right) \geqslant \tau_{2} \boldsymbol{\alpha}(n) r^{n} \text {, }
$$

where

$$
E_{S}=h_{S} \circ \mu_{r}\left(U^{-}\right) \cap D
$$

and $r=2^{-1} \operatorname{diam} S$. Set

$$
E(i+1)=\cup\left\{E_{S}: S \in G_{i}\right\}
$$

and

$$
G_{i+1}=\cup\left\{\left\{h_{S} \circ \mu_{r}(T): T \in F\right\}: S \in G_{i}\right\} .
$$

One sees easily that (a)-(d) above are satisfied. Since, in particular, the sets $E(i)$ are pairwise disjoint, one has

$$
\begin{aligned}
\mathfrak{L}^{n}(D \cap \operatorname{Int} C) & \geqslant \sum_{i=1}^{m+1} \mathcal{L}^{n}(E(i)) \geqslant \tau_{2} \sum_{i=0}^{m}\left(\frac{\tau_{1}}{2}\right)^{i} \mathcal{L}^{n}\left(\cup G_{0}\right) \\
& \geqslant \mathcal{L}^{n}(C) \tau_{1} \tau_{2} \sum_{i=0}^{m}\left(\frac{\tau_{1}}{2}\right)^{i}>\lambda \mathcal{L}^{n}(C) .
\end{aligned}
$$

The above shows that if $C_{1}, C_{2}, C_{2}, \ldots \in \mathcal{C}_{\mathrm{e}}$ with $\lim _{i} C_{i}=C$ in the Hausdorff distance topology, then

$$
\mathfrak{L}^{n}(C) \leqslant \lim _{i} \inf ^{\mathfrak{L}^{n}}\left(C_{i}\right) .
$$

Since $\mathfrak{L}^{n}(C)=\lim _{\sigma \rightarrow 0+} \mathfrak{L}^{n} \circ e_{\sigma}(C)$ and $d\left(C, C_{i}\right)<\sigma$ implies $C_{i} \subset e_{\sigma}(C)$, one sees that

$$
\mathfrak{L}^{n}(C) \geqslant \lim \sup \mathfrak{L}^{n}\left(C_{i}\right) \text {. }
$$

(5) Assume $C \in \mathcal{C}$ and $C_{1}, C_{2}, C_{3}, \ldots \in \mathcal{C}(B, L)$ with $\lim _{i} C_{i}=C$ in the Hausdorff distance topology. One checks that, for $j=1,2,3, \ldots$,

$$
D_{j}=C \cup \bigcup_{i>j} C_{i} \in \mathcal{C}
$$

and notes, by [RE, Lemma 7A], that $D_{j} \in \mathcal{C}(B, L)$. Also one has

$$
D_{1} \supset D_{2} \supset D_{3} \supset \cdots \text { and } \bigcap_{j} D_{j}=C \text {, }
$$

so it follows from [RE, Lemma 21A] that $C \in \mathcal{C}(B, L)$.

(6) Select $Y \in \mathcal{C}(B, L)$ with $\varrho^{n} \circ e_{e}(Y)<2 \mu_{e}(B, L)$, which by (1) we can do. Then there exists an integer $i$ with

$$
1 \leqslant i \leqslant 1+2 \boldsymbol{\alpha}(n)^{-1} \varepsilon^{-n} \mu_{e}(B, L)
$$

such that

$$
Y \cap \mathbf{S}^{n-1}(0, r+2 \varepsilon i)=\varnothing
$$

where $r=d(\{0\}, B)$. 
By a direct sum theorem for Čech homology (see [ES, I, 13.2 and X]) one has

$$
Y \cap \mathbf{B}^{n}(0, r+2 \varepsilon i) \in \mathcal{C}(B, L) .
$$

Conclusion (6) now follows from (2)-(5).

\section{The one dimensional case.}

4.1. Lemma. Assume $m$ is a positive integer $\gamma \in \mathbf{R}^{+},\left\{C, C_{1}, C_{2}, C_{3}, \ldots\right\} \subset$ $\mathcal{C},\{\varepsilon(1), \varepsilon(2), \varepsilon(3), \ldots\} \subset \mathbf{R}^{+}$with $\lim _{i} \varepsilon(i)=0,\{\delta(1), \delta(2), \delta(3), \ldots\} \subset \mathbf{R}^{+}$ $\cup\{0\}$ with $\lim _{i} \delta(i)=0$, and that, for each $i=1,2,3, \ldots, C_{i}$ consists of no more than $m$ connected components and $\delta(i)=d\left(C, C_{i}\right)$.

(1) There are no more than $m$ connected components in $C$.

(2) If one has

$$
\mathcal{L}^{n} \circ e_{\mathrm{e}(i)}\left(C_{i}\right) \leqslant \gamma[\varepsilon(i)]^{n-1}
$$

for $i=1,2,3, \ldots$, then $\mathcal{H}^{1}(C)$ is finite and $C$ is 1-rectifiable.

(3) If $\mathcal{H}^{1}(C)$ is finite, then one has

$$
\mathcal{H}^{1}(C) \leqslant \liminf _{i} \mathcal{L}^{n} \circ e_{\varepsilon(i)}\left(C_{i}\right) \boldsymbol{\alpha}(n-1)^{-1} \varepsilon(i)^{1-n} .
$$

Proof. (1) Conclusion (1) is clear.

(2) By [FH, 2.8.14] there exists a positive integer $b$ such that, for each $i=1$, $2,3, \ldots$, there exist disjointed families, $F_{1}, F_{2}, F_{3}, \ldots, F_{b}$, of closed balls of radius $\varepsilon(i)$ centered in $C_{i}$ so that $C_{i} \subset \cup\left(\cup_{j} F_{j}\right)$. The hypothesis of (2) implies

$$
\operatorname{card}\left(F_{j}\right) \leqslant \gamma \boldsymbol{\alpha}(n)^{-1} \varepsilon(i)^{-1},
$$

for $j=1,2,3, \ldots, b$. Set $L_{i}$ equal to the union of all the line segments the endpoints of which are the centers of a pair of intersecting balls in $\cup_{j} F_{j}$. One observes that

$$
\mathcal{H}^{1}\left(L_{i}\right) \leqslant 2 \cdot 3^{n} b^{2} \gamma \boldsymbol{\alpha}(n)^{-1}
$$

because, for each $r \in \mathbf{R}^{+}$, each closed ball in $\mathbf{R}^{n}$ of radius $r$ intersects no more than $3^{n}$ disjointed balls of radius $r$. Observe also that each $L_{i}$ consists of no more than $m$ connected components, $L_{i, 1}, L_{i, 2}, L_{i, 3}, \ldots, L_{i, m(i)}$, and that the $L_{i}$ converge to $C$ in the Hausdorff distance topology.

Set

$$
K=\bigcup_{j=1}^{m}\{t: 2 j \leqslant t \leqslant 2 j+1\} .
$$

For $i=1,2,3 \ldots$ and $j=1,2,3, \ldots, m(i)$, by [EH, Theorem 2] there exists a function $f_{i, j}:\{t: 2 j \leqslant t \leqslant 2 j+1\} \rightarrow \mathbf{R}^{n}$ with $f_{i, j}(\{t: 2 j<t<2 j+1\})=L_{i, j}$ and $\operatorname{Lip}\left(f_{i, j}\right) \leqslant 2 \mathcal{F}^{1}\left(L_{i, j}\right)$. We then define $f_{i}: K \rightarrow \mathbf{R}^{n}$ by setting

$$
f_{i}(t)=f_{i, j}(t), \quad \text { if } j=1,2,3, \ldots, m(i) \text { and } 2 j<t \leqslant 2 j+1 \text {, }
$$


and

$$
f_{i}(t)=f_{i, m(i)}(2 m(i)+1), \quad \text { if } 2 m(i)+1<t .
$$

We have

$$
f_{i}(K)=L_{i}
$$

and

$$
\begin{aligned}
\operatorname{Lip}\left(f_{i}\right) & \leqslant \sup \left\{\operatorname{diam} L_{i}\right\} \cup\left\{2 \mathcal{H}^{1}\left(L_{i, j}\right): j=1,2,3, \ldots, m(i)\right\} \\
& \leqslant \sup \left\{\operatorname{diam} L_{i}, 2 \mathcal{H}^{1}\left(L_{i}\right)\right\} .
\end{aligned}
$$

The above estimate for $\mathcal{H C}^{1}\left(L_{i}\right)$ gives a uniform bound for $\operatorname{Lip}\left(f_{i}\right)$. Since $\cup_{i} L_{i} \cup C$ is compact, we see that a subsequence of $f_{1}, f_{2}, f_{3}, \ldots$ converges to a Lipschitzian function $f$. It is clear then that $f(K)=C$.

(3) By (1) and [EH, Theorem 2], $C$ is 1-rectifiable. Let $C^{\prime}$ be the set of $c \in C$ such that $\Theta^{1}\left(\mathcal{F C}^{1}\llcorner C, c)=1\right.$ and $\operatorname{Tan}^{1}\left(\mathcal{F C}^{1}\llcorner C, c)\right.$ is a 1 dimensional vectorsubspace of $\mathbf{R}^{n}$. By [FH, 3.2.19] we have $\mathcal{H}^{1}(C)=\mathcal{H}^{1}\left(C^{\prime}\right)$.

Let $0<\sigma<3^{-1}$ be arbitrary. We will show that for each $c \in C^{\prime}$ there exists $r_{0}(c)>0$ so that if $0<r<r_{0}(c)$, then

$$
\begin{aligned}
\lim _{i} \inf & \mathcal{L}^{n}\left[e_{e(i)}\left(C_{i}\right) \cap \mathbf{B}^{n}(c, r)\right] \boldsymbol{\alpha}(n-1)^{-1} \varepsilon(i)^{1-n} \\
> & 2\left(2+6^{-1} \sigma\right)^{-1}\left(1-2 \sigma-3 \sigma^{2}\right)^{1 / 2} \mathcal{H}^{1}\left[C^{\prime} \cap \mathbf{B}^{n}(c, r)\right]
\end{aligned}
$$

holds. Once this is established, conclusion (3) is obtained as follows: By [FH, 2.8.15], we can find a disjointed family of closed balls, $\left\{\mathbf{B}^{n}\left(c_{j}, r_{j}\right): j=1,2\right.$, $3, \ldots\}$, such that $c_{j} \in C^{\prime}, 0<r_{j}<r_{0}\left(c_{j}\right)$, for $j=1,2,3, \ldots$, and

$$
\mathcal{K}^{1}\left[C^{\prime} \cap \bigcup_{j} \mathbf{B}^{n}\left(c_{j}, r_{j}\right)\right]=\mathcal{H}^{1}\left(C^{\prime}\right)
$$

then we have

$$
\begin{aligned}
& \lim _{i} \inf \mathcal{L}^{n} \circ e_{\varepsilon(i)}\left(C_{i}\right) \boldsymbol{\alpha}(n-1)^{-1} \varepsilon(i)^{1-n} \\
& >\liminf _{i} \mathfrak{L}^{n}\left[e_{e(i)}\left(C_{i}\right) \cap \bigcup_{j} \mathbf{B}^{n}\left(c_{j}, r_{j}\right)\right] \boldsymbol{\alpha}(n-1)^{-1} \varepsilon(i)^{1-n} \\
& =\lim _{i} \inf \sum_{j} \mathfrak{L}^{n}\left[e_{\varepsilon(i)}\left(C_{i}\right) \cap \mathbf{B}^{n}\left(c_{j}, r_{j}\right)\right] \boldsymbol{\alpha}(n-1)^{-1} \varepsilon(i)^{1-n} \\
& \geqslant \sum_{j} \lim _{i} \inf \mathcal{L}^{n}\left[e_{e(i)}\left(C_{i}\right) \cap \mathbf{B}^{n}\left(c_{j}, r_{j}\right)\right] \boldsymbol{\alpha}(n-1)^{-1} \varepsilon(i)^{1-n} \\
& \geqslant 2\left(2+6^{-1} \sigma\right)^{-1}\left(1-2 \sigma-3 \sigma^{2}\right)^{1 / 2} \sum_{j} \mathcal{H}^{1}\left[C^{\prime} \cap \mathbf{B}^{n}\left(c_{j}, r_{j}\right)\right] \\
& =2\left(2+\sigma^{-1} \sigma\right)^{-1}\left(1-2 \sigma-3 \sigma^{2}\right)^{1 / 2} \mathcal{H}^{1}(C) .
\end{aligned}
$$


Now, fix $c \in C^{\prime}$. To simplify the notation we suppose $c=0$ and that $\operatorname{Tan}^{1}\left(\mathcal{K}^{1}\llcorner C, c)\right.$ is spanned by $\mathbf{e}_{1}$. Set

$$
\begin{aligned}
C^{\prime \prime} & =C \sim\{x:\{x\} \text { is a connected component of } C\}, \\
r_{1} & =\operatorname{dist}\left(C^{\prime \prime}, C \sim C^{\prime \prime}\right), \\
r_{2} & =\inf \left\{2^{-1} \operatorname{diam} K: K \text { is a connected component of } C^{\prime \prime}\right\} .
\end{aligned}
$$

Note that $C^{\prime} \subset C^{\prime \prime}$, and if $x \in C^{\prime \prime}$ and $0<r<r_{2}$, then, by [FH, 2.10.12],

$$
\mathcal{H}^{1}\left[C^{\prime} \cap \mathbf{B}^{n}(x, r)\right] \geqslant r \text {. }
$$

It is clear that we can pick $r_{3}>0$ so that $0<r<r_{3}$ implies

$$
\mathcal{H}^{1}\left[C^{\prime} \cap \mathbf{B}^{n}(0, r)\right] \leqslant\left(2+6^{-1} \sigma\right) r
$$

and

$$
\mathcal{H}^{1}\left[C^{\prime} \cap \mathbf{B}^{n}(0, r) \cap\left\{x:|\mathbf{q}(x)| \leqslant(2+\sigma)^{-1} \sigma r\right\}\right]>\left(2-6^{-1} \sigma\right) r,
$$

where q: $\mathbf{R}^{n} \rightarrow \mathbf{R}^{n-1}$ is defined by

$$
\mathbf{q}\left(x_{1}, x_{2}, x_{3}, \ldots, x_{n}\right)=\left(x_{2}, x_{3}, \ldots, x_{n}\right) \text {. }
$$

Set

$$
r_{4}=\inf \left\{r_{1}, 2 r_{2} \sigma^{-1}, 2 r_{3}(1+\sigma)^{-1}\right\} \text {. }
$$

We claim that if $0<r<r_{4}$, then

$$
C \cap \mathbf{B}^{n}(0, r) \cap\{x:|\mathbf{q}(x)|>\sigma r\}=\varnothing .
$$

To see this, suppose on the contrary that

$$
x \in C \cap \mathbf{B}^{n}(0, r) \cap\{x:|\mathbf{q}(x)|>\sigma r\} .
$$

Since $r<r_{1}$, we have $x \in C^{\prime \prime}$, and thus, since $2^{-1} \sigma r<r_{2}$,

$$
\mathcal{H}^{1}\left[C^{\prime} \cap \mathbf{B}^{n}\left(x, 2^{-1} \sigma r\right)\right] \geqslant 2^{-1} \sigma r .
$$

Also, we have

$$
\mathbf{B}^{n}\left(x, 2^{-1} \sigma r\right) \subset \mathbf{B}^{n}\left[0,2^{-1} r(2+\sigma)\right] \cap\left\{x:|\mathbf{q}(x)|>2^{-1} \sigma r\right\} .
$$

It follows, since $2^{-1} r(2+\sigma)<r_{3}$, that

$$
\mathcal{H}^{1}\left[C^{\prime} \cap \mathbf{B}^{n}\left(0,2^{-1} r(2+\sigma)\right)\right] \geqslant 2^{-1} \sigma r+\left(2-6^{-1} \sigma\right) 2^{-1} r(2+\sigma)
$$

and

$$
\mathcal{H}^{1}\left[C^{\prime} \cap \mathbf{B}^{n}\left(0,2^{-1} r(2+\sigma)\right)\right] \leqslant\left(2+6^{-1} \sigma\right) 2^{-1} r(2+\sigma),
$$

which is a contradiction.

Next we note, using the fact that $\operatorname{Tan}^{1}\left(\mathcal{H}^{1}\llcorner C, 0)\right.$ is the vector space spanned by $e_{1}$, that there is $0<r_{0}<r_{4}$ so that if $0<r<r_{0}$ and

$$
-r\left(1-\sigma^{2}\right)^{1 / 2}<t<r\left(1-\sigma^{2}\right)^{1 / 2},
$$

then

$$
C \cap \mathbf{B}^{n}(0, r) \cap\left\{x: x \cdot \mathbf{e}_{1}=t\right\} \neq \varnothing .
$$


Now fix $0<r<r_{0}$. Consider a positive integer $i$ such that $\delta(i)<\sigma r$ and $\varepsilon(i)<\sigma r$. Set

$$
a=r\left(1-2 \sigma-3 \sigma^{2}\right)^{1 / 2}
$$

We claim that if

$$
\begin{gathered}
-a \leqslant t_{1}<t_{2} \leqslant a, \quad t_{2}-t_{1}>2 \delta(i), \\
C_{i} \cap \mathbf{B}^{n}(0,(1-\sigma) r) \cap\left\{x: x \cdot \mathbf{e}_{1}=t_{1}\right\}=\varnothing,
\end{gathered}
$$

and

$$
C_{i} \cap \mathbf{B}^{n}(0,(1-\sigma) r) \cap\left\{x: x \cdot \mathbf{e}_{1}=t_{2}\right\}=\varnothing
$$

hold, then $\mathbf{B}^{n}(0,(1-\sigma) r) \cap\left\{x: t_{1} \leqslant x \cdot \mathbf{e}_{1} \leqslant t_{2}\right\}$ will contain a connected component of $C_{i}$. To see this, it will suffice to show

$$
C_{i} \cap \mathbf{S}^{n-1}(0,(1-\sigma) r) \cap\left\{x:-a \leqslant x \cdot \mathbf{e}_{1} \leqslant a\right\}=\varnothing
$$

and

$$
C_{i} \cap \mathbf{B}^{n}(0,(1-\sigma) r) \cap\left\{x: t_{1} \leqslant x \cdot \mathbf{e}_{1} \leqslant t_{2}\right\} \neq \varnothing .
$$

Suppose

$$
x \in C_{i} \cap \mathbf{S}^{n-1}(0,(1-\sigma) r) \cap\left\{x:-a<x \cdot \mathbf{e}_{1} \leqslant a\right\},
$$

then $|\mathbf{q}(x)| \geqslant 2 \sigma r$. Also, there is $x^{\prime} \in C \cap \mathbf{B}^{n}(x, \delta(i))$, so

$$
x^{\prime} \in C \cap \mathbf{B}^{n}(0, r) \cap\{x:|\mathbf{q}(x)|>\sigma r\},
$$

a contradiction. On the other hand, there does exist

$$
w \in C \cap\left\{x:|\mathbf{q}(x)| \leqslant \sigma r, x \cdot \mathbf{e}_{1}=2^{-1}\left(t_{1}+t_{2}\right)\right\}
$$

and, thus,

$$
w^{\prime} \in C_{i} \cap \mathbf{B}^{n}(w, \delta(i)) .
$$

One notes then that

$$
\left|w^{\prime}\right| \leqslant(1-\sigma) r \text { and } t_{1}<w^{\prime} \cdot \mathbf{e}_{1}<t_{2}
$$

It follows from the above that

$$
\begin{aligned}
& \mathcal{L}^{n}\left[e_{\varepsilon(i)}\left(C_{i}\right)\right.\left.\cap \mathbf{B}^{n}(0, r)\right] \boldsymbol{\alpha}(n-1)^{-1} \varepsilon(i)^{1-n} \\
& \geqslant 2\left(1-2 \sigma-3 \sigma^{2}\right)^{1 / 2} r-2(m+1) \delta(i),
\end{aligned}
$$

and hence we have (\#).

4.2. Theorem. Assume $B \subset \mathbf{R}^{n}$ is finite, $L \subset H_{0}(B) \cap \operatorname{ker}[\operatorname{aug}(B)]$ is a subgroup, $\{\varepsilon(1), \varepsilon(2), \varepsilon(3), \ldots\} \subset \mathbf{R}^{+}$with $\lim _{i} \varepsilon(i)=0$, and $\left\{C_{1}, C_{2}, C_{3}, \ldots\right\}$ $\subset \mathcal{C}$ with $C_{i} \in \Re_{\varepsilon(i)}(B, L)$ for each $i=1,2,3, \ldots$

(1) For each $i=1,2,3, \ldots$, there exists $C_{i}^{\prime} \subset C_{i}$ such that $C_{i}^{\prime} \in \mathcal{C}(B, L)$, $e_{e(i)}\left(C_{i}^{\prime}\right)=e_{e(i)}\left(C_{i}\right)$, and each connected component of $C_{i}^{\prime}$ contains a point of $B$. 
(2) For each $i=1,2,3, \ldots$, one has

$$
\begin{aligned}
\mathcal{L}^{n} \circ e_{\varepsilon(i)}\left(C_{i}\right) \leqslant & \operatorname{diam}(B) \cdot \operatorname{card}(B) \cdot \boldsymbol{\alpha}(n-1) \varepsilon(i)^{n-1} \\
& +\operatorname{card}(B) \cdot \boldsymbol{\alpha}(n) \varepsilon(i)^{n} .
\end{aligned}
$$

(3) There exists $r \in \mathbf{R}^{+}$such that $C_{i} \subset \mathbf{B}(0, r)$ for each $i=1,2,3, \ldots$

(4) There exist $C \in \mathcal{C}$, which is 1-rectifiable, and a subsequence $i(1), i(2)$, $i(3), \ldots$ of $1,2,3, \ldots$ so that $C_{i(j)}$ converges to $C$ in the Hausdorff distance topology as $j \rightarrow \infty$.

(5) For each $C$ as in (4), one has $C \in \mathcal{C}(B, L)$ and

$$
\mathcal{H}^{1}(C)=\inf \left\{\mathcal{H}^{1}(X): X \in \mathcal{C}(B, L)\right\}<\infty .
$$

Proof (1) One sets $C_{i}^{\prime}$ equal to the union of all connected components of $C_{i}$ which contain a point of $B$. Conclusion (1) then follows from a direct sum theorem for Čech homology (see [ES, I, 13.2 and X] and [RE, Lemma 21A]).

(2) Conclusion (2) follows from [RE, Lemma 2A], applied as in the proof of $3(1)$, and an obvious estimate.

(3) Conclusion (3) follows from the construction in the proof of 3(6) where we use (2) to bound $\mu_{e(i)}(B, L)$.

(4) It follows from (3) and 3(2) that a subsequence $C_{i(1)}, C_{i(2)}, C_{i(3)}, \ldots$ converges to $C$. One notes also that $C_{i(j)}^{\prime}$ converges to $C$ in the Hausdorff distance topology, so (2) and 4.1(2) imply that $C$ is 1-rectifiable.

(5) Let $C$ be as in (4), then $C \in \mathcal{C}(B, L)$ by 3(5). Suppose $X \in \mathcal{C}(B, L)$ with $\mathcal{H}^{1}(X)<\infty$. As in the proof of (1), one sees that there exists $Y \subset X$ with $Y \in \mathcal{C}(B, L)$ such that $Y$ has finitely many connected components. By [EH, Theorem 2] and [FH, 3.2.39], one sees that

$$
\mathcal{H}^{1}(Y)=\lim _{\varepsilon \rightarrow 0^{+}} \mathcal{L}^{n} \circ e_{\varepsilon}(Y) \boldsymbol{\alpha}(n-1)^{-1} \varepsilon^{1-n} .
$$

By (1) and 4.1(3), since $C_{i}^{\prime} \in \mathfrak{N}_{\mathrm{e}(i)}(B, L)$, one concludes

$$
\mathcal{H}^{1}(C) \leqslant \mathcal{H}^{1}(Y) \leqslant \mathcal{H}^{1}(X) \text {. }
$$

\section{Polyhedral complexes.}

5.1. Defintions. (1) A 0 polyhedron is a point of $\mathbf{R}^{n}$; for each integer $m>1$, an $m$ polyhedron is a bounded, relatively open, nonempty subset of an $m$ dimensional affine subspace of $\mathbf{R}^{n}$ the boundary of which is the closure of the union of finitely many $m-1$ polyhedra.

(2) For each nonnegative integer $m$, an $m$ polyhedral complex is a subset, $P$, of $\mathbf{R}^{n}$ together with a decomposition of $P$ into a finite union of $0,1,2, \ldots, m$ polyhedra such that for each $l=1,2,3, \ldots, m$ the boundary of each $l$ polyhedron of the decomposition is contained in the union of the 0,1 , $2, \ldots, l-1$ polyhedra of the decomposition; for $l=0,1,2, \ldots, m$, denote by $P_{l}$ the union of the $0,1,2, \ldots, l$ polyhedra of the decomposition and set $\mathscr{P}^{l}(P)=\mathcal{H}^{l}\left(P_{l}\right)$; for each integer $l<0$ or $l>m$ set $P_{l}=\varnothing$ and $\mathscr{P}^{l}(P)=0$. 
5.2. Notation. In 5.3 we will use the following notation. For each $c \in \mathbf{R}^{+}$, set

$$
K(0,2, c)=\sup \left\{2 c^{-1} n^{1 / 2}, 1\right\} \text { and } K(n, m, c)=2 n^{1 / 2},
$$

whenever $m=3,4,5, \ldots, n$. For each $c \in \mathbf{R}^{+}$, each $m=3,4,5, \ldots, n$, and each $l=n-1, n-2, n-3, \ldots, 0$, set

$$
K(l, m, c)=w\left[1+2(u+1)(m+1)^{2} 2^{(n+1) / 2}\right]
$$

where

$$
u=K\left(0, m-1,\left[(m+1) 2^{(n+2) / 2} c^{1-m}\right]^{-1 /(m-2)}\right)
$$

and

$$
w=K\left(l+1, m, c\left[u(m+1) 2^{(n+2) / 2}+1\right]^{-1 /(m-1)}\right) .
$$

Finally, for each $c \in \mathbf{R}^{+}$and each $m=2,3,4, \ldots, n$, set

$$
K(m, c)=K(0, m, c) \text {. }
$$

5.3. TheOREM. (1) For each $m$ polyhedral complex $P(0<m<n)$ and each $\varepsilon \in \mathbf{R}^{+}$,

$$
\mathcal{L}^{n} \circ e_{\varepsilon}(P) \leqslant \sum_{i} \mathscr{P}^{i}(P) \boldsymbol{\alpha}(n-i) \varepsilon^{n-i} .
$$

(2) For each $c \in \mathbf{R}^{+}$, each 1 polyhedral complex $A$, and each $d \in \mathbf{R}^{+}$with

$$
d \geqslant c^{\mathcal{P l}^{1}}(A)
$$

there exists a 2 polyhedral complex $P$ with

$$
P \in \mathcal{C}\left[A, H_{1}(A)\right], \quad P \subset \operatorname{Cv}(A) \cap e_{d K(2, c)}(A),
$$

and, for each integer $i$,

$$
\mathscr{P}^{i}(P) \leqslant K(2, c) \sum_{j>-1} \mathscr{P}^{i+j}(A) d^{-j} .
$$

(3) Let $l$ and $m$ be integers with $0 \leqslant l \leqslant n$ and $3 \leqslant m \leqslant n$. Fix a family $\left\{\Pi_{1}, \Pi_{2}, \Pi_{3}, \ldots, \Pi_{l}\right\}$ of pairwise orthogonal $n-1$ dimensional affine subspaces of $\mathbf{R}^{n}$. For each $c \in \mathbf{R}^{+}$, each $m-1$ polyhedral complex $A$, and each $d \in \mathbf{R}^{+}$with

and such that

$$
d \geqslant c\left[\mathscr{P}^{m-1}(A)\right]^{1 /(m-1)}
$$

$$
\operatorname{dist}\left(a, \Pi_{i}\right) \leqslant d,
$$

for each $i=1,2,3, \ldots, l$ and each $a \in A$, there exists an $m$ polyhedral complex $P$ with

$$
P \in \mathcal{C}\left[A, H_{m-1}(A)\right], \quad P \subset \operatorname{Cv}(A) \cap e_{d K(l, m, c)}(A),
$$

and, for each integer $i$,

$$
\mathscr{P}^{i}(P) \leqslant K(l, m, c) \sum_{j>-1} \mathscr{P}^{i+j}(A) d^{-j} .
$$


(4) For each $m=2,3,4, \ldots, n$, each $c \in \mathbf{R}^{+}$, each $m-1$ polyhedral complex $A$, and each $d \in \mathbf{R}^{+}$with

$$
d \geqslant c\left[\mathcal{P}^{m-1}(A)\right]^{1 /(m-1)},
$$

there exists an $m$ polyhedral complex $P$ with

$$
P \in \mathcal{C}\left[A, H_{m-1}(A)\right], \quad P \subset \operatorname{Cv}(A) \cap e_{d K(m, c)}(A),
$$

and, for each integer $i$,

$$
\mathscr{P}^{i}(P) \leqslant K(m, c) \sum_{j>-1} \mathscr{P}^{i+j}(A) d^{-j} .
$$

Proof. (1) Conclusion (1) is clear.

(2) Let $A$ be a nonempty 1 polyhedral complex. Let $\left\{\Pi_{1}, \Pi_{2}, \Pi_{3}, \ldots, \Pi_{n}\right\}$ be a family of pairwise orthogonal $n-1$ dimemsional affine subspaces of $\mathbf{R}^{n}$. For each $i=1,2,3, \ldots, n$ choose $v_{i} \in \mathbf{S}^{n-1}(0,1)$ perpendicular to $\Pi_{i}$ and, for each $0 \leqslant s \leqslant 2 c^{-1} d$, set

$$
\Pi_{i}(s)=\left\{x+\left(s+2 \lambda c^{-1} d\right) v_{i}: x \in \Pi_{i}, \lambda \in \mathbf{Z}\right\} .
$$

For each $i=1,2,3, \ldots, n$, by [FH, 2.10.11] one has

$$
\int_{0}^{2 c^{-1} d} \mathcal{H}^{0}\left[A \cap \Pi_{i}(s)\right] d \mathfrak{L}_{s}^{1} \leqslant \mathcal{H}^{1}(A)=\mathscr{P}^{1}(A),
$$

so one can choose $0<s_{i}<2 c^{-1} d$ such that $A \cap \Pi_{i}\left(s_{i}\right)=\varnothing$. One sees that, for some integer $r$,

$$
A=A(1) \cup A(2) \cup A(3) \cup \cdots \cup A(r)
$$

where, for each $i=1,2,3, \ldots, r, A(i) \neq \varnothing$ is a 1 polyhedral complex, there is an open cube $C(i)$ with side length $2 c^{-1} d$ such that $A(i) \subset C(i)$, and, further, the family $\{C(i)\}_{i=1,2,3, \ldots, r}$ is disjointed. For each $i=1,2,3, \ldots, r$ choose $x_{i} \in A(i)_{0}$. One can make $\left\{x_{i}\right\} * A(i)$ a 2 polyhedral complex such that

$$
\begin{gathered}
\mathscr{P}^{2}\left[\left\{x_{i}\right\} * A(i)\right] \leqslant n^{1 / 2} c^{-1} d \mathscr{P}^{1}[A(i)], \\
\mathscr{P}^{1}\left[\left\{x_{i}\right\} * A(i)\right] \leqslant 2 n^{1 / 2} c^{-1} d \mathscr{P}^{0}[A(i)]+\mathscr{P}^{1}[A(i)],
\end{gathered}
$$

and

$$
\mathscr{P}^{0}\left[\left\{x_{i}\right\} * A(i)\right]=\mathscr{P}^{0}[A(i)]
$$

Set

$$
P=\bigcup_{i}\left[\left\{x_{i}\right\} \nVdash A(i)\right]
$$

and note that $P$ can be made a 2 polyhedral complex such that

$$
\begin{gathered}
\mathscr{P}^{2}(P) \leqslant n^{1 / 2} c^{-1} d^{1}(A)<K(2, c)^{\mathcal{P}^{1}}(A) d, \\
\mathscr{P}^{1}(P) \leqslant 2 n^{1 / 2} c^{-1} d \mathscr{P}^{0}(A)+\mathcal{P}^{1}(A) \leqslant K(2, c)\left[\mathscr{P}^{0}(A) d+\mathscr{P}^{1}(A)\right],
\end{gathered}
$$


and

$$
\mathscr{P}^{0}(P)=\mathscr{P}^{0}(A) \leqslant K(2, c)^{\mathscr{P}^{0}}(A) .
$$

By [RE, Lemma $3 \mathrm{~A}], P \in \mathcal{C}\left[A, H_{1}(A)\right]$ and it is clear that

$$
P \subset \operatorname{Cv}(A) \cap e_{d K(2, c)}(A) .
$$

(3) First we consider (3) in the case $l=n$. Let $A$ be a nonempty $m-1$ polyhedral complex. Set $\{y\}=\cap{ }_{i} \Pi_{i}$ and choose $x \in A_{0}$. Notice $A \subset$ $\mathbf{B}^{n}\left(y, n^{1 / 2} d\right)$ so $A \subset \mathbf{B}^{n}\left(x, 2 n^{1 / 2} d\right)$. Set $P=\{x\} * A$ and notice that $P$ can be made an $m$ polyhedral complex such that for each integer $i>0$,

$$
\begin{aligned}
\mathscr{P}^{i}(P) & \leqslant 2 n^{1 / 2} i^{-1} \mathscr{P}^{i-1}(A) d+\mathscr{P}^{i}(A) \\
& \leqslant K(n, m, c)\left[\mathscr{P}^{i-1}(A) d+\mathscr{P}^{i}(A)\right]
\end{aligned}
$$

and

$$
\mathscr{P}^{0}(P)=\mathscr{P}^{0}(A) \leqslant K(n, m, c) \mathscr{P}^{0}(A) .
$$

By [RE, Lemma 2A], the conclusion of (3) follows in case $l=n$.

Set

$$
N=\{(2,0)\} \cup\{(m, l): 3 \leqslant m \leqslant n, 0 \leqslant l \leqslant n\}
$$

and define the relation $\prec$ on $N$ by setting $\left(m^{\prime}, l^{\prime}\right) \prec(m, l)$ if and only if

(a) $m^{\prime}<m$, or

(b) $m^{\prime}=m$ and $l^{\prime}>l$.

The set $N$ is well ordered by $\prec$ and $(2,0)$ is the smallest element. Say that $(m, l) \in N^{\prime}$ if $(m, l) \in N$ and the conclusion of (3) holds for that pair of integers. By (2) we have $(2,0) \in N^{\prime}$ and, by the preceding paragraph, $(m, n) \in N^{\prime}$ for each $m=3,4,5, \ldots, n$. We will prove (3) by induction: Fix $(m, l) \in N$ and suppose $\left(m^{\prime}, l^{\prime}\right) \in N^{\prime}$ for each $\left(m^{\prime}, l^{\prime}\right)<(m, l)$. We must show $(m, l) \in N^{\prime}$. We may assume $3 \leqslant m$ and $l \leqslant n-1$.

Let $A$ be a nonempty $m-1$ polyhedral complex. Let $\Pi$ be an $n-1$ dimensional affine subspace of $\mathbf{R}^{n}$ perpendicular to $\Pi_{1}, \Pi_{2}, \Pi_{3}, \ldots$, and $\Pi_{l}$ and choose $v \in \mathbf{S}^{n-1}(0,1)$ perpendicular to $\Pi$. Set

$$
\Pi(s)=\{x+(s+\lambda d) v: x \in \Pi, \lambda \in \mathbf{Z}\} .
$$

By [FH, 2.10.25 and 3.2.13] one has

$$
\int_{0}^{d} \mathcal{H}^{i-1}\left[A_{i} \cap \Pi(s)\right] d \mathscr{L}_{s}^{1} \leqslant 2^{(n+2) / 2 \mathscr{P}^{i}}(A)
$$

(one uses the fact

$$
\begin{aligned}
\boldsymbol{\alpha}(r+2) \boldsymbol{\alpha}(r+3)^{-1} & =\left[(r+3)(r+2)^{-1}\right] \boldsymbol{\alpha}(r) \boldsymbol{\alpha}(r+1)^{-1} \\
& <2 \boldsymbol{\alpha}(r) \boldsymbol{\alpha}(r+1)^{-1}
\end{aligned}
$$

for $r \in \mathbf{R}^{+}$), so one can choose $0<s_{0}<d$ such that no polyhedron of $A$ is 
contained in $\Pi\left(s_{0}\right)$ and

$$
\mathcal{H}^{i-1}\left[A_{i} \cap \Pi\left(s_{0}\right)\right] \leqslant(m+1) 2^{(n+2) / 2} \mathscr{\rho P}^{i}(A) d^{-1}
$$

for $i=1,2,3, \ldots, m-1$. For each integer $r$, set

$$
D(r)=A \cap\left\{x+\left(s_{0}+r d\right) v: x \in \Pi\right\} .
$$

Notice that $D(r)$ can be made an $m-2$ polyhedral complex with

$$
d \geqslant\left[(m+1) 2^{(n+2) / 2} c^{1-m}\right]^{-1 /(m-2)}\left\{\mathscr{P}^{m-2}[D(r)]\right\}^{1 /(m-2)} .
$$

Notice also that $D(r)=\varnothing$ for all but finitely many $r \in \mathbf{Z}$.

By the induction hypothesis $\left((m-1,0) \in N^{\prime}\right)$, for each integer $r$, there exists an $m-1$ polyhedral complex $B(r)$ such that

$$
\begin{aligned}
& B(r) \in \mathcal{C}\left\{D(r), H_{m-2}[D(r)]\right\}, \\
& B(r) \subset \operatorname{Cv}[D(r)] \cap e_{d u}[D(r)],
\end{aligned}
$$

and, for each integer $i$,

$$
\mathscr{P}^{i}[B(r)] \leqslant u \sum_{j>-1} \mathscr{P}^{i+j}[D(r)] d^{-j},
$$

where

$$
u=K\left(0, m-1,\left[(m+1) 2^{(n+2) / 2} c^{1-m}\right]^{-1 /(m-2)}\right) .
$$

For each integer $r$ set

$$
\begin{aligned}
A(r)= & B(r) \cup B(r+1) \cup A \\
& \cap\left\{x+\left[s_{0}+(r+t) d\right] v: x \in \Pi, 0 \leqslant t<1\right\} ;
\end{aligned}
$$

notice that each point of $A(r)$ lies within $d$ of $l+1$ pairwise orthogonal $n-1$ dimensional affine subspaces of $\mathbf{R}^{n}$ and that $A(r)$ can be made an $m-1$ polyhedral complex with

$$
\begin{aligned}
\mathscr{P}^{m-1}[A(r)] & \leqslant \mathscr{P}^{m-1}[B(r)]+\mathscr{P}^{m-1}[B(r+1)]+\mathcal{P}^{m-1}(A) \\
& \leqslant\left[u(m+1) 2^{(n+2) / 2}+1\right] \mathcal{P}^{m-1}(A) .
\end{aligned}
$$

By the induction hypothesis $\left((m, l+1) \in N^{\prime}\right)$, for each integer $r$, there exists $P(r)$ an $m$ polyhedral complex such that

$$
\begin{aligned}
& P(r) \in \mathcal{C}\left\{A(r), H_{m-1}[A(r)]\right\}, \\
& P(r) \subset \operatorname{Cv}[A(r)] \cap e_{d w}[A(r)],
\end{aligned}
$$

and, for each integer $i$,

$$
\mathcal{P}^{i}[P(r)] \leqslant w \sum_{j>-1} \mathcal{P}^{i+j}[A(r)] d^{-j},
$$

where

$$
w=K\left(l+1, m, c\left[u(m+1) 2^{(n+2) / 2}+1\right]^{-1 /(m-1)}\right) .
$$


Set $P=\cup{ }_{r} P(r)$. Notice that by [RE, Lemma 13A] $P \in C\left[A, H_{m-1}(A)\right]$, and that $P \subset \operatorname{Cv}(A) \cap e_{d(u+w)}(A)$. We can make $P$ a polyhedral complex such that for each integer $i$,

$$
\begin{aligned}
\mathscr{P}^{i}(P)= & \sum_{r} \mathscr{P}^{i}[P(r)] \leqslant w \sum_{r} \sum_{j>-1} \mathscr{P}^{i+j}[A(r)] d^{-j} \\
\leqslant & w \sum_{j>-1}\left\{\mathscr{P}^{i+j}(A)+2 \sum_{r} \mathscr{P}^{i+j}[B(r)]+2 \sum_{r} \mathscr{P}^{i+j}[D(r)]\right\} d^{-j} \\
\leqslant & w \sum_{j>-1}\left\{\mathscr{P}^{i+j}(A)+2 u \sum_{r} \sum_{h>-1} \mathcal{P}^{i+j+h}[D(r)] d^{-h}\right. \\
& \left.+2 \sum_{r} \mathscr{P}^{i+j}[D(r)]\right\} d^{-j} \\
\leqslant & w \sum_{j>-1} \mathscr{P}^{i+j}(A) d^{-j} \\
& +2 u w(m+1) 2^{(n+2) / 2} \sum_{j>-1} \sum_{h>-1} \mathscr{P}^{i+j+h+1}(A) d^{-j-h-1} \\
& +2 w(m+1) 2^{(n+2) / 2} \sum_{j>-1} \mathscr{P P}^{i+j+1}(A) d^{-j-1} \\
\leqslant & w\left[1+2(u+1)(m+1)^{2} 2^{(n+2) / 2}\right] \sum_{j>-1} \mathscr{P}^{i+j}(A) d^{-j},
\end{aligned}
$$

which proves (3).

(4) Conclusion (4) is a special case of (3).

5.4. Preliminaries. (1) Set $\mathbf{A}=\mathbf{R}^{n} \cap\{x: \mathbf{m}(x) \leqslant 1\}$.

(2) Fix $\theta \in \mathbf{R}^{+}$. For each $m \in\{0,1,2, \ldots, n\}, \sigma_{m}$ will be as in [FH, 4.2.6], and, for each $a \in \mathbf{A}$, we set

$$
\begin{aligned}
\Psi(m, a) & =\tau_{a} \circ \sigma_{m} \circ \tau_{-a}, \\
\Psi(m, a, \theta) & =\mu_{\theta} \circ \Psi(m, a) \circ \mu_{1 / \theta} .
\end{aligned}
$$

(3) Let $P$ be a $k-1$ polyhedral complex. For $\mathfrak{L}^{n}$ almost every $a \in \mathbf{A}$ we have

$$
\tau_{-a}(P) \cap \mathbf{W}_{n-k}^{\prime \prime}=\varnothing \text { and } \tau_{-a} \circ \mu_{1 / \theta}(P) \cap \mathbf{W}_{n-k}^{\prime \prime}=\varnothing,
$$

so $\Psi(k-1, a)$ and $\Psi(k-1, a, \theta)$ are defined on $P$. Restricting our attention to such $a \in \mathbf{A}$, we set

$$
\begin{aligned}
& H(P, a)=\bigcup_{m=k}^{n}\{t \Psi(m, a)(x)+(1-t) \Psi(m-1, a)(x): \\
&0 \leqslant t \leqslant 1, x \in P\}
\end{aligned}
$$

and

$$
\begin{array}{r}
H(P, a, \theta)=\bigcup_{m=k}^{n}\{t \Psi(m, a, \theta)(x)+(1-t) \Psi(m-1, a, \theta)(x): \\
0 \leqslant t \leqslant 1, x \in P\} .
\end{array}
$$


We would like to pick a decomposition of $P$ so that if $L$ is an $l$ polyhedron of the decomposition, then $\Psi(m, a)(L)$ is again an $l$ polyhedron $(m=k-1$, $k, k+1, \ldots, n)$. Then $H(P, a)$ can be made a $k$ polyhedral complex and estimates for $\mathscr{P}^{i}[H(P, a)]$ can be proved. To do this, the decomposition of $P$ will have to depend on the choice of $a \in \mathbf{A}$. Thus we are lead to formulate the following definitions.

(4) Let $r$ be an integer, $0 \leqslant r \leqslant n-1$. Let $I$ be a family of $r$ dimensional affine subspaces of $\mathbf{R}^{n}$; $\Pi$ will be called an affine $r$ family if the elements of $\boldsymbol{\Pi}$ are parallel to each other and only finitely many elements of $\boldsymbol{\Pi}$ intersect any compact subset of $\mathbf{R}^{n}$. Finally, $\{\varnothing\}$ will be the only affine $(-1)$ family.

(5) Let $P \subset \mathbf{R}^{n}$ be an $m$ polyhedral complex and let $\Pi$ be an affine $r$ family. Define the $m$ polyhedral complex $P * \Pi$ by letting the $l$ polyhedra of the decomposition of $P * \Pi$ consist of

(a) the connected components of $L \cap\left(\mathbf{R}^{n} \sim \cup \mathbf{I}\right)$ where $L$ is an $l$ polyhedron of the decomposition of $P$ (each component is to be taken as a separate polyhedron),

(b) $L \cap \Pi$ where $\Pi \in \Pi, L$ is a polyhedron of the decomposition of $P$, and $L \cap \Pi$ is $l$ dimensional.

(6) If $\Pi_{1}$ is an affine $r_{1}$ family and $\Pi_{2}$ is an affine $r_{2}$ family, then $\Pi_{1} \wedge \Pi_{2}$ will denote $\left\{\Pi_{1} \cap \Pi_{2}: \Pi_{1} \in \Pi_{1}\right.$ and $\left.\Pi_{2} \in \Pi_{2}\right\}$. We note that $\Pi_{1} \wedge \Pi_{2}$ is an affine $r_{3}$ family for some $r_{3} \leqslant \inf \left\{r_{1}, r_{2}\right\}$ and that if $P$ is an $m$ polyhedral complex, then, for each integer $l$,

$$
\left[\left(P * \Pi_{1}\right) * \Pi_{2}\right]_{l} \subset\left[P * \Pi_{1}\right]_{l} \cup\left[P * \Pi_{2}\right]_{l} \cup\left[P *\left(\Pi_{1} \wedge \Pi_{2}\right)\right]_{l}
$$

(7) For each integer $r$ with $0 \leqslant r \leqslant n-1$, let $\mathbf{F}_{r}^{\prime}$ be the collection of $r$ dimensional affine subspaces each of which contains $r+1$ affinely independent points of $\mathbf{A} \cap \mathbf{Z}^{n}$, set

$$
\mathbf{F}_{r}=\left\{\boldsymbol{\tau}_{z}(\Pi): z \in \mathbf{Z}_{n}^{n}, \Pi \in F_{r}^{\prime}\right\},
$$

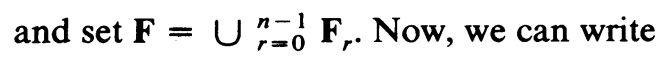

$$
\mathbf{F}=\boldsymbol{\Pi}_{1}^{\prime} \cup \boldsymbol{\Pi}_{2}^{\prime} \cup \boldsymbol{\Pi}_{3}^{\prime} \cup \cdots \cup \boldsymbol{\Pi}_{s^{\prime}}^{\prime}
$$

where $\Pi_{i}^{\prime}$ is an affine $r_{i}$ family $(0 \leqslant r \leqslant n-1)$ for each $i$ and the $\Pi_{i}^{\prime}$ are pairwise disjoint. Let $\Pi_{1}, \Pi_{2}, \Pi_{3}, \ldots, \Pi_{s}$ be an enumeration of

$$
\left\{\boldsymbol{\Pi}_{\lambda(1)}^{\prime} \wedge \boldsymbol{\Pi}_{\lambda(2)}^{\prime} \wedge \boldsymbol{\Pi}_{\lambda(3)}^{\prime} \wedge \cdots \wedge \boldsymbol{\Pi}_{\lambda(j)}^{\prime}: 1 \leqslant j \leqslant s^{\prime}, \lambda \in \Lambda\left(s^{\prime}, j\right)\right\} .
$$

(8) Let $P$ be a $k-1$ polyhedral complex. For each $a \in \mathbf{A}$, set

$$
\left.P^{\prime}=\tau_{a}\left[\left(\ldots\left(\tau_{-a}(P) * \Pi_{1}^{\prime}\right) * \Pi_{2}^{\prime}\right) * \ldots\right) * \Pi_{s^{\prime}}^{\prime}\right]
$$

$P^{\prime}$ is $P$ with a new decomposition, and if $L$ is an $l$ polyhedron of $P^{\prime}$, then $\Psi(m, a)(L)$ is an $l$ polyhedron for $m=k-1, k, k+1, \ldots, n$ (provided $\left.\tau_{-a}(P) \cap \mathbf{W}_{n-k}^{\prime \prime}=\varnothing\right)$. Thus $H(P, a)=H\left(P^{\prime}, a\right)$ can be made a $k$ polyhedral complex for $\mathcal{L}^{n}$ almost every $a \in \mathbf{A}$. 
5.5. THEOREM. (1) If $V$ is an $r$ dimensional linear subspace of $\mathbf{R}^{n}$, then there is $\lambda \in \Lambda(n, r)$ so that $\mathbf{p}_{\lambda}$ (see $\left.[\mathbf{F H}, 1.7 .4]\right)$ is one-to-one when restricted to $V$ and

$$
\left|\left(\mathbf{p}_{\lambda} \mid V\right)^{-1}\left(\mathbf{e}_{i}\right)\right| \leqslant\left(\begin{array}{l}
n \\
r
\end{array}\right)
$$

holds for $i=1,2,3, \ldots, r$.

(2) There exists $\gamma \in \mathbf{R}^{+}$such that if $P$ is a $k-1$ polyhedral complex, then one has

$$
\int_{\mathrm{A}} \int_{\left(\tau_{a}(P) * \Pi_{j}\right)_{l}}\left(u_{k-1}\right)^{-l} d \mathcal{H}^{l} d \mathfrak{L}_{a}^{n}<\gamma \sum_{i=l}^{k-1} \mathscr{P}^{i}(P)
$$

for $0<l<k-1$ and $1 \leqslant j \leqslant s$; here $u_{k-1}$ is as in [FH, 4.2.6].

(3) There exists $\gamma(n, k) \in \mathbf{R}^{+}$such that, for each $k-1$ polyhedral complex $P$,

(a) $H(P, a)$ can be made a $k$ polyhedral complex so as to satisfy

$$
\int_{\mathbf{A}} \mathscr{P}^{l+1}[H(P, a)] d \mathfrak{L}_{a}^{n} \leqslant \gamma(n, k) \sum_{i=l}^{k-1} \mathscr{P}^{i}(P),
$$

(b) for each $0<\varepsilon<1$ one has

$$
\int_{\mathrm{A}} \mathfrak{L}^{n} \circ e_{\varepsilon}[H(p, a)] d \mathfrak{L}_{a}^{n} \leqslant 6(k+1) \gamma(n, k) \sum_{i} \mathscr{P}^{i}(P) \varepsilon^{n-i-1},
$$

(c) for each $0<\varepsilon<\theta$ one has

$$
\int_{\mathbf{A}} \mathfrak{L}^{n} \circ e_{\varepsilon}[H(P, a, \theta)] d \mathcal{L}_{a}^{n} \leqslant 6(k+1) \gamma(n, k) \theta \sum_{i} \mathscr{P}^{i}(P) \varepsilon^{n-i-1} .
$$

Proof. (1) Let $\mathbf{v}_{1}, \mathbf{v}_{2}, \mathbf{v}_{3}, \ldots, \mathbf{v}_{r}$ be an orthonormal basis for $V$. Write

$$
\mathbf{v}_{i}=\left(v_{i 1}, v_{i 2}, v_{i 3}, \ldots, v_{i n}\right)
$$

for $i=1,2,3, \ldots, r$. By the Lagrange identity (see [BR, p. 49, Theorem 1]), we see there is $\lambda \in \Lambda(n, r)$ so that

$$
\left|\operatorname{det}\left[\left(v_{i \lambda(j)}\right)_{1<i, j<r}\right]\right| \geqslant\left(\begin{array}{c}
n \\
r
\end{array}\right)^{-1} .
$$

Define $f: \mathbf{R}^{r} \rightarrow V$ by setting $f\left(\mathbf{e}_{i}\right)=\mathbf{v}_{i}$ for $i=1,2,3, \ldots, r$. Then we have $\left|\operatorname{det}\left(\mathbf{p}_{\lambda} \circ f\right)\right| \geqslant\left(\begin{array}{c}n \\ r\end{array}\right)^{-1}$ and $\left|\mathbf{p}_{\lambda} \circ f(x)\right| \leqslant|x|$ for all $x \in \mathbf{R}^{r}$. It follows that, for $x \in \mathbf{R}^{r}$,

$$
|x|^{-1}\left|\mathbf{p}_{\lambda} \circ f(x)\right| \geqslant\left|\operatorname{det}\left(\mathbf{p}_{\lambda} \circ f\right)\right| \geqslant\left(\begin{array}{l}
n \\
r
\end{array}\right)^{-1},
$$

which implies conclusion (1), since $\left(\mathbf{p}_{\lambda} \mid V\right)^{-1}=f \circ\left(\mathbf{p}_{\lambda} \circ f\right)^{-1}$.

(2) Fix a $k-1$ polyhedral complex $P$ and integers $l$, $j$ with $0<l<k-1$, $1<j<s$.

Let $L$ be an $l$ polyhedron of $P$. Using [FH, 4.2.7(1)], we have 


$$
\begin{aligned}
& \int_{\mathbf{A}} \int_{\tau_{-a}(L) \cap\left(\mathbf{R}^{n} \sim \cup \mathbf{\Pi}_{j}\right)}\left(u_{k-1}\right)^{-l} d \mathcal{H}^{l} d \mathcal{L}_{a}^{n} \\
& \quad \leqslant \int_{\mathbf{A}} \int_{\tau_{-a}(L)}\left(u_{k-1}\right)^{-l} d \mathcal{H}^{l} d \mathcal{L}_{a}^{n} \leqslant \int_{\mathbf{A}} \int_{\tau_{-a}(L)}\left(u_{k-1}\right)^{1-k} d \mathcal{H}^{l} d \mathcal{L}_{a}^{n} \\
& \quad=\int_{\mathbf{A}} \int_{L}\left(u_{k-1} \circ \tau_{-a}\right)^{1-k} d \mathcal{H}^{l} d \mathcal{L}_{a}^{n} \leqslant 2^{n}\left(\begin{array}{c}
n \\
k-1
\end{array}\right) \mathcal{H}^{l}(L) .
\end{aligned}
$$

To proceed with the proof we must consider the following situation: Let $V$ be an $r$ dimensional linear subspace of $\mathbf{R}^{n}$ where $r \geqslant n+l+1-k$, let $\Pi$ be a translate of $V$, and let $X$ be an $\left(\mathcal{H}^{l}, l\right)$ rectifiable Suslin set contained in $\Pi$. Suppose also that $\mathbf{p}: \mathbf{R}^{n} \rightarrow \mathbf{R}^{r}$ defined by

$$
\mathbf{p}\left(x_{1}, x_{2}, x_{3}, \ldots, x_{n}\right)=\left(x_{1}, x_{2}, x_{3}, \ldots, x_{r}\right)
$$

is one-to-one when restricted to $V$ and

$$
c \geqslant \prod_{i=1}^{r}\left|(\mathbf{p} \mid V)^{-1}\left(\mathbf{e}_{i}\right)\right|
$$

We have

$$
c \geqslant\left[\operatorname{ap} J_{r}(\mathbf{p} \mid V)(v)\right]^{-1}, \quad \text { for } v \in V
$$

and

$$
c \geqslant\left[\operatorname{ap} J_{l}(\mathbf{p} \mid X)(x)\right]^{-1}, \quad \text { for } \mathcal{H}^{l} \text { almost all } x \in X .
$$

Set $m=k+r-n-1$. Let $u_{m}^{\prime}$ be defined as $u_{m}$ is in [FH, 4.2.6] but with $n$ replaced by $r$. Using [FH, 3.2.22] and the fact that $u_{k-1}(x)>u_{m}^{\prime} \circ \mathbf{p}(x)$, we obtain

$$
\begin{aligned}
& \int_{V \cap \mathbf{B}^{n}(0, \sqrt{n})} \int_{X}\left(u_{k-1} \circ \tau_{-v}\right)^{-l} d \mathcal{H}^{l} d \mathcal{H}_{v}^{r} \\
& \quad \leqslant c^{2} \int_{\mathbf{p}\left[V \cap \mathbf{B}^{n}(0, \sqrt{n})\right]} \int_{\mathbf{p}(X)}\left(u_{m}^{\prime} \circ \tau_{-w}\right)^{-l} d \mathcal{H}^{l} d \mathcal{H}_{w}^{r} .
\end{aligned}
$$

Now using [FH, 4.2.7(1)], we have

$$
\begin{aligned}
\int_{\mathbf{p}\left[V \cap \mathbf{B}^{n}(0, \sqrt{n})\right]} \int_{\mathbf{p}(X)}\left(u_{m}^{\prime} \circ \tau_{-w}\right)^{-l} d \mathcal{H}^{d} d \mathcal{H}_{w}^{r} \\
\leqslant \int_{\mathbf{p}\left[V \cap \mathbf{B}^{n}(0, \sqrt{n})\right]} \int_{\mathbf{p}(X)}\left(u_{m}^{\prime} \circ \tau_{-w}\right)^{-m} d \mathcal{H}^{d} d \mathcal{H}_{w}^{r} \\
\leqslant(n+1)^{r} \int_{\mathbf{p}(\mathbf{A})} \int_{\mathbf{p}(X)}\left(u_{m}^{\prime} \circ \tau_{-w}\right)^{-m} d \mathcal{H}^{C} d \mathcal{H}_{w} \\
\leqslant(n+1)^{r} 2^{r}\left(\begin{array}{c}
r \\
m
\end{array}\right) \mathcal{H}^{d}(X) .
\end{aligned}
$$


Thus we conclude

$$
\begin{aligned}
& \int_{V \cap \mathbf{B}^{n}(0, \sqrt{n})} \int_{X}\left(u_{k-1} \circ \tau_{-v}\right)^{-l} d \mathcal{H}^{d} d \mathcal{H}_{v} \\
& \leqslant c^{2}(n+1)^{r} 2^{r}\left(\begin{array}{c}
r \\
k+r-n-1
\end{array}\right) \mathcal{H C}^{d}(X) .
\end{aligned}
$$

Let $L$ be an $l^{\prime}$ polyhedron of $P$ and suppose $\Pi_{j}$ is an affine $r$ family. Fix $\Pi \in \Pi_{j}$. Let $V$ be the $r$ dimensional linear subspace of $\mathbf{R}^{n}$ parallel to $\Pi$ and let $W$ be the orthogonal complement of $V$. If $l^{\prime}+r-n<0$, then $\tau_{-a}(L) \cap$ $\Pi=\varnothing$ for $\varrho^{n}$ almost every $a \in \mathbf{A}$. If $l^{\prime}+r-n \geqslant 0$ then, for $\mathcal{L}^{n}$ almost every $a \in \mathbf{A}$, either $\tau_{-a}(L) \cap \Pi=\varnothing$ or $\tau_{-a}(L) \cap \Pi$ is an $l^{\prime}+r-n$ polyhedron. We are trying to estimate the integral of $\left(u_{k-1}\right)^{-l}$ over the $l$ polyhedra of $\tau_{-a}(P) * \Pi_{j}$, thus only $r$ such that $l=l^{\prime}+r-n$ are of interest. So we assume $r=n+l-l^{\prime}$ and notice $n+l-l^{\prime} \geqslant n+l+1-k$.

Both sides of the inequality in (2) are invariant under a linear isometry of $\mathbf{R}^{n}$ which permutes the standard basis vectors. So, by (1), we may assume that p, as above, is one-to-one when restricted to the $V$ of the preceding paragraph and we may take

$$
c=\left(\begin{array}{l}
n \\
r
\end{array}\right)^{r} \text {. }
$$

Then, by the above and [FH, 2.10.25], we have

$$
\begin{aligned}
\int_{\mathbf{A}} & \int_{\tau_{-a}(L) \cap \Pi}\left(u_{k-1}\right)^{-l} d \mathcal{H}^{l} d \mathcal{L}_{a}^{n} \\
\leqslant & \int_{W \cap \mathbf{B}^{n}(0, \sqrt{n})} \int_{V \cap \mathbf{B}^{n}(0, \sqrt{n})} \int_{\tau_{-w}(L) \cap \Pi}\left(u_{k-1} \circ \tau_{-v}\right)^{-l} d \mathcal{H}^{l} d \mathcal{H}_{v}^{r} d \mathcal{H}_{w}^{n-r} \\
\leqslant & \left(\begin{array}{c}
n \\
r
\end{array}\right)^{2 r}(n+1)^{r} 2^{r}\left(\begin{array}{c}
r \\
k+r-n-1
\end{array}\right) \\
& \cdot \int_{W \cap \mathbf{B}^{n}(0, \sqrt{n})} \mathcal{H}\left[\tau_{-w}(L) \cap \Pi\right] d \mathcal{H}_{w}^{n-r} \\
\leqslant & \left(\begin{array}{c}
n \\
r
\end{array}\right)^{2 r}(n+1)^{r} 2^{r}(k+r-n-1) \boldsymbol{\alpha}(l) \boldsymbol{\alpha}(n-r) \boldsymbol{\alpha}\left(l^{\prime}\right)^{-1} \\
& \cdot \mathcal{H}^{\prime}[\underbrace{\left.r \cap \tau_{w}(\Pi)\right] .}_{w \in W \cap \mathbf{B}^{n}(0, \sqrt{n})}
\end{aligned}
$$

Now, define an equivalence relation on $\Pi_{j}$ by

$$
\Pi_{1} \approx \Pi_{2} \text { if and only if } \Pi_{1}=\tau_{z} \Pi_{2} \text { for some } z \in \mathbf{Z}_{n}^{n} \text {. }
$$

Then $\boldsymbol{\Pi}_{j}$ consists of finitely many, say $c_{j}$, equivalence classes. It follows that

$$
\begin{aligned}
& \int_{\mathbf{A}} \int_{\tau_{-a}(L) \cap \Pi_{j}}\left(u_{k-1}\right)^{-1} d \mathcal{H C} d \mathscr{L}_{a}^{n} \\
& \leqslant\left(\begin{array}{c}
n \\
r
\end{array}\right)^{2 r}(n+1)^{r} 2^{r}\left(\begin{array}{c}
r \\
k+r-n-1
\end{array}\right) \boldsymbol{\alpha}(l) \boldsymbol{\alpha}(n-r) \boldsymbol{\alpha}\left(l^{\prime}\right)^{-1} \\
& \quad \cdot c_{j} \operatorname{card}\left\{z \in \mathbf{Z}_{n}^{n}:|z| \leqslant 2 \sqrt{n}\right\} \mathcal{H}^{\prime}(L) .
\end{aligned}
$$


Conclusion (2) now follows.

(3a) We use the decomposition of $H(P, a)$ indicated in 5.4(8). Assuming that $\tau_{-a}(P) \cap \mathbf{W}_{n-k}^{\prime \prime}=\varnothing$, we see that any $l+1$ polyhedron is either of the form

(i) $\Psi(m, a)(L)$, for some $m=n, n-1, n-2, \ldots, k-1$ and some $l+1$ polyhedron $L$ of $P^{\prime}$ (here $0 \leqslant l+1 \leqslant k-1$ ), or of the form

(ii) $\{t \Psi(m, a)(x)+(1-t) \Psi(m-1, a)(x): 0<t<1, x \in L\}$, for some $m=n, n-1, n-2, \ldots, k$ and some $l$ polyhedron $L$ of $P^{\prime}$ (here $1<l+1$ $<k)$.

Now, we have, for $n \geqslant m \geqslant k-1$,

$$
\begin{aligned}
\mathcal{H}^{l+1}[\Psi(m, a)(L)] & \leqslant \int_{\tau_{-a}(L)}\left\|D \sigma_{m}\right\|^{l+1} d \mathcal{H}^{l+1} \\
& \leqslant(1+m)^{l+1} \int_{\tau_{-a}(L)}\left(u_{m}\right)^{-(l+1)} d \mathcal{H}^{l+1} \\
& \leqslant(1+m)^{l+1} \int_{\tau_{-a}(L)}\left(u_{k-1}\right)^{-(l+1)} d \mathcal{H}^{l+1},
\end{aligned}
$$

where we have used [FH, 4.2.6]. Next, notice that

$$
\begin{aligned}
\mathcal{H}^{l+1}[\{t \Psi(m, a) & (x)+(1-t) \Psi(m-1, a)(x): 0<t<1, x \in L\}] \\
& <(l+1)^{-1} \cdot n^{1 / 2} \cdot \mathcal{H}[\Psi(m-1, a)(L)] \\
& <(l+1)^{-1} \cdot n^{1 / 2} \cdot m^{l} \int_{\tau_{-a}(L)}\left(u_{k-1}\right)^{-l} d \mathcal{H}^{l},
\end{aligned}
$$

where we have used the preceding observation with $l+1$ replaced by $l$ and $m$ replaced by $m-1$. So, by (2), we have

$$
\begin{aligned}
& \int_{\mathbf{A}} \mathscr{P}^{l+1}[H(P, a)] d \mathcal{L}_{a}^{n} \\
& \leqslant \sum_{m=k-1}^{n}(1+m)^{l+1} \int_{\mathbf{A}} \int_{\tau_{-a}\left(P_{i+1}^{\prime}\right)}\left(u_{k-1}\right)^{-(l+1)} d \mathcal{C}^{C+1} d \mathcal{L}_{a}^{n} \\
& \quad+\sum_{m=k}^{n}(l+1)^{-1} \cdot n^{1 / 2} \cdot m^{l} \int_{\mathbf{A}} \int_{\tau_{-a}\left(P_{l}^{\prime}\right)}\left(u_{k-1}\right)^{-l} d \mathcal{C}^{C} d \mathcal{L}_{a}^{n} \\
& \leqslant n(n+1)^{k-1} \sum_{j=1}^{s} \int_{\mathbf{A}} \int_{\left(\tau_{-a}(P) * \Pi_{j}\right)_{l+1}}\left(u_{k-1}\right)^{-(l+1)} d \mathcal{H}^{l+1} d \mathcal{L}_{a}^{n} \\
& \quad+n^{(2 k+1) / 2} \sum_{j=1}^{s} \int_{\mathbf{A}} \int_{\left(\tau_{-a}(P) * \Pi_{j}\right)_{l+1}}\left(u_{k-1}\right)^{-l} d \mathcal{H}^{l} d \mathcal{L}_{a}^{n} \\
& \leqslant n(n+1)^{k-1} \cdot s \cdot \gamma \sum_{i=l+1}^{k-1} \mathscr{P}(P)+n^{(2 k+1) / 2} \cdot s \cdot \gamma \sum_{i=l}^{k-1} \mathscr{P}(P) \\
& \leqslant {\left[n(n+1)^{k-1}+n^{(2 k+1) / 2}\right] \cdot s \cdot \gamma \sum_{i=l}^{k-1} \mathcal{P}(P), }
\end{aligned}
$$


where we have also used

$$
P_{l+1}^{\prime} \subset \bigcup_{j=1}^{s}\left(P * \Pi_{j}\right)_{l+1} \text { and } P_{l}^{\prime} \subset \bigcup_{j=1}^{s}\left(P * \Pi_{j}\right)_{l} \text {. }
$$

(3b) By 5.3(1), (3a) above, and the fact that $\alpha(i)<6$ for each positive integer $i$, one has

$$
\begin{aligned}
\int_{\mathbf{A}} \mathfrak{L}^{n} \circ e_{\varepsilon}[H(P, a)] d \mathfrak{L}_{a}^{n} & \leqslant 6 \sum_{l=-1}^{k-1} \varepsilon^{n-l-1} \int_{\mathbf{A}} \mathscr{P}^{l+1}[H(P, a)] d \mathfrak{L}_{a}^{n} \\
& \leqslant 6 \sum_{l=-1}^{k-1} \gamma(n, k) \varepsilon^{n-l-1} \sum_{i=l}^{k-1} \mathcal{P}^{i}(P) \\
& \leqslant 6 \gamma(n, k) \sum_{l=-1}^{k-1} \sum_{i=l}^{k-1} \varepsilon^{n-i-1} \mathscr{P}^{i}(P) \\
& \leqslant 6(k+1) \gamma(n, k) \sum_{i} \mathscr{P}^{i}(P) \varepsilon^{n-i-1}
\end{aligned}
$$

(3c) Conclusion (3c) follows from (3b) since $Q=\mu_{1 / \theta}(P)$ is a $k-1$ polyhedral complex with

$$
\mathcal{P}^{i}(Q)=\theta^{-i} \mathscr{P}^{i}(P),
$$

for $i=0,1,2, \ldots, k-1$, and

$$
e_{\varepsilon}[H(p, a, \theta)]=\mu_{\theta} \circ e_{\varepsilon / \theta}[H(Q, a)] .
$$

\section{Lower bound on density.}

6.1. Preliminaries. For use in 6.2 we assume

(1) $k \geqslant 2$,

(2) $B \in \mathcal{C}$ and $L \subset H_{k-1}(B)$ is a subgroup,

(3) $\{\varepsilon(1), \varepsilon(2), \varepsilon(3), \ldots\} \subset \mathbf{R}^{+}$with $\lim _{i} \varepsilon(i)=0$,

(4) $C_{i} \in \mathfrak{N}_{\mathrm{e}(i)}(B, L)$ for each $i=1,2,3, \ldots$

For $i=1,2,3, \ldots$, define the measure $g_{i}$ over $\mathbf{R}^{n}$ by setting

$$
\mathscr{g}_{i}(A)=\left[\boldsymbol{\alpha}(n-k) \varepsilon(i)^{n-k}\right]^{-1} \cdot \mathfrak{L}^{n}\left[e_{\varepsilon(i)}\left(C_{i}\right) \cap A\right]
$$

for each $A \subset \mathbf{R}^{n}$ and the function

$$
l_{i}: \mathbf{R}^{n} \times \mathbf{R}^{+} \rightarrow \mathbf{R}
$$

by setting

$$
l_{i}(x, r)=\left[\boldsymbol{\alpha}(n-k) \varepsilon(i)^{n-k}\right]^{-1} \cdot \mathcal{H}^{n-1}\left[e_{e(i)}\left(C_{i}\right) \cap \mathbf{S}^{n-1}(x, r)\right] .
$$

6.2. TheOREM. There exists $\Gamma_{1} \in \mathbf{R}^{+}$, which does not depend on $B, L,\{\varepsilon(i)\}$, or $\left\{C_{i}\right\}$, such that the following hold:

(1) For each $i=1,2,3, \ldots$, if $x \in \mathbf{R}^{n}$ and $r \geqslant \varepsilon(i)$ with $\mathbf{B}^{n}(x, r) \cap B=\varnothing$ and $C_{i} \cap \mathbf{U}^{n}(x, r) \neq \varnothing$, then one has

$$
g_{i}\left[\mathbf{B}^{n}(x, r)\right] \leqslant \Gamma_{1}\left[l_{i}(x, r)\right]^{k /(k-1)} .
$$


(2) For each $i=1,2,3, \ldots$, if $x \in C_{i}$ and $r \geqslant \varepsilon(i)$ with $\mathbf{B}^{n}(x, r) \cap B=\varnothing$, then one has

$$
\mathscr{g}_{i}\left[\mathbf{B}^{n}(x, r)\right] \geqslant k^{-k} \Gamma_{1}^{1-k}[r-\varepsilon(i)]^{k}
$$

(3) If $B$ is $k-1$ rectifiable, then one has

$$
\lim \sup \mathscr{G}_{i}\left(\mathbf{R}^{n}\right) \leqslant k^{-1} \cdot \operatorname{diam}(B) \cdot \mathcal{H}^{k-1}(B) .
$$

(4) If $B$ is $k-1$ rectifiable, then there exists a subsequence $i(1), i(2)$, $i(3), \ldots$ of $1,2,3, \ldots$, a compact set $C \subset \mathbf{R}^{n}$, and a Radon measure $f$ over $\mathbf{R}^{n}$ with compact support such that $\lim _{j} C_{i(j)}=C$ in the Hausdorff distance topology and $\mathcal{G}$ is the weak limit of $\mathscr{G}_{i(j)}$ as $j \rightarrow \infty$. For any such subsequence, $C \subset \mathbf{R}^{n}$, and measure $\mathcal{G}$ one has

(a) $C \in \mathcal{C}(B, L)$,

(b) if $x \in C, r \in \mathbf{R}^{+}$, with $\mathbf{B}^{n}(x, r) \cap B=\varnothing$, then

$$
\mathcal{G}\left[\mathbf{B}^{n}(x, r)\right] \geqslant k^{-k} \Gamma_{1}^{1-k} r^{k},
$$

(c) $C \sim B \subset \operatorname{spt}(g) \subset C$,

(d) $\mathscr{H}^{k}(C)<\infty$.

Proof. (1) By [FH, 2.8.14] there exists a positive integer $b$ such that for each triple $(i, x, r)$ as in (1) there exist disjointed families, $F_{1}, F_{2}, F_{3}, \ldots, F_{b}$, of closed balls of radius $2^{-1} \varepsilon(i)$ centered in $C_{i} \cap \mathbf{S}^{n-1}(x, r)$ so that

$$
C_{i} \cap \mathbf{S}^{n-1}(x, r) \subset \cup\left(\bigcup_{j} F_{j}\right) \text {. }
$$

It is clear that there exists $c \in \mathbf{R}^{+}$such that for each $y \in \mathbf{S}^{n-1}(x, r)$,

$$
\mathcal{F}^{n-1}\left[\mathbf{S}^{n-1}(x, r) \cap \mathbf{B}^{n}\left(y, 2^{-1} \varepsilon(i)\right)\right] \geqslant c \varepsilon(i)^{n-1} \text {. }
$$

Hence, setting $\mathscr{F}=\cup_{j} F_{j}$ and $l=l_{i}(x, r)$, one sees that

$$
\operatorname{card}(\mathscr{F}) \leqslant \alpha(n-k) b c^{-1} l \varepsilon(i)^{1-k}
$$

Choose a collection of open balls, $\mathcal{G}$, such that there is a one-to-one and onto function $f: \mathcal{F} \rightarrow \mathcal{G}$ with $f\left[\mathbf{B}^{n}\left(y, 2^{-1} \varepsilon(i)\right)\right]=\mathbf{U}^{n}(y, s)$ for some $2^{-1} \varepsilon(i)<s<$ $\varepsilon(i)$ and

$$
f\left[\mathbf{B}^{n}\left(y, 2^{-1} \varepsilon(i)\right)\right] \cap f\left[\mathbf{B}^{n}\left(z, 2^{-1} \varepsilon(i)\right)\right]=\varnothing
$$

if and only if

$$
\mathbf{B}^{n}\left(y, 2^{-1} \varepsilon(i)\right) \cap \mathbf{B}^{n}\left(a, 2^{-1} \varepsilon(i)\right)=\varnothing
$$

whenever $\left[\mathbf{B}^{n}\left(y, 2^{-1} \varepsilon(i)\right), \mathbf{B}^{n}\left(z, 2^{-1} \varepsilon(i)\right)\right] \in \mathscr{F} \times \mathcal{F}$. For each $D \in \mathcal{G}$ one sees, as in the proof of $4.1(2)$, that

$$
\operatorname{card}\{E: E \in \mathcal{G}, D \cap E \neq \varnothing\}<3^{n} b .
$$


Notice that $\mathbf{N}\left[\mathcal{G}, C_{i} \cap \mathbf{S}^{n-1}(x, r)\right]$ can obviously be considered as a polyhedral complex and set

$$
A=\mathbf{N}\left[\mathcal{G}, C_{i} \cap \mathbf{S}^{n-1}(x, r)\right]_{k-1} .
$$

Observe that, for $j=0,1,2, \ldots, k-1$,

$$
\mathscr{P}^{j}(A) \leqslant \boldsymbol{\alpha}(j)\left(3^{n} b\right)^{k-1} \boldsymbol{\alpha}(n-k) b c^{-1} \cdot l \varepsilon(i)^{j+1-k},
$$

so, in particular,

$$
\begin{aligned}
l^{1 /(k-1)} \geqslant & {\left[\boldsymbol{\alpha}(k-1)\left(3^{n} b\right)^{k-1} \alpha(n-k) b c^{-1}\right]^{-1 /(k-1)} } \\
& \cdot\left[\mathscr{P}^{k-1}(A)\right]^{1 /(k-1)} .
\end{aligned}
$$

One applies 5.3(4) with $m$ replaced by $k, c$ replaced by

$$
\left[\boldsymbol{\alpha}(k-1)\left(3^{n} b\right)^{k-1} b \boldsymbol{\alpha}(n-k) c^{-1}\right]^{-1 /(k-1)},
$$

and $d=l^{1 /(\mathrm{k}-1)}$ to obtain $p \in \mathcal{C}\left[A, H_{k-1}(A)\right]$ a $k$ polyhedral complex with

$$
\begin{aligned}
\mathscr{P}^{m}(P) & \leqslant \gamma_{1} \sum_{j>-1} \mathcal{P}^{m+j}(A) l^{-j /(k-1)} \\
& \leqslant \gamma_{2} \sum_{j=-1}^{k-1-m} l^{(k-1-j) /(k-1)} \varepsilon(i)^{m+j+1-k}
\end{aligned}
$$

for $m=0,1,2, \ldots, k$, where $\gamma_{1}$ and $\gamma_{2}$ are appropriate constants. By 5.3(1) one has

$$
\mathcal{L}^{n} \circ e_{\varepsilon(i)}(P) \leqslant \gamma_{2} \sum_{m=0}^{k} \sum_{j=-1}^{k-1-m} \alpha(n-m) l^{(k-1-j) /(k-1)} \varepsilon(i)^{n+j+1-k} .
$$

Since $C_{i} \cap \mathbf{U}^{n}(x, r) \neq \varnothing$, we conclude, by a direct sum theorem for Čech homology, that $C_{i} \cap \mathbf{S}^{n-1}(x, r) \neq \varnothing$, thus

$$
l \geqslant c \boldsymbol{\alpha}(n-k)^{-1} \varepsilon(i)^{k-1},
$$

and it follows that

$$
\mathcal{L}^{n} \circ e_{\varepsilon(i)}(P) \leqslant \gamma_{3} l^{k /(k-1)} \varepsilon(i)^{n-k},
$$

for an appropriate $\gamma_{3} \in \mathbf{R}^{+}$. Set

$$
C=\left[C_{i} \sim \mathbf{B}^{n}(x, r)\right] \cup e_{2 \varepsilon(i)}\left[C_{i} \cap \mathbf{S}^{n-1}(x, r)\right] \cup P,
$$

so, by 8.4(1), $C \in \mathcal{C}(B, L)$. Observe that

$$
\begin{aligned}
\mathcal{L}^{n} \circ e_{\varepsilon(i)} \circ e_{2 \varepsilon(i)}[ & \left.C_{i} \cap \mathbf{S}^{n-1}(x, r)\right] \\
& \leqslant \boldsymbol{\alpha}(n) 4^{n} \boldsymbol{\alpha}(n-k) b c^{-1} l \varepsilon(i)^{n-k+1}
\end{aligned}
$$

and

$$
\begin{aligned}
\mathfrak{L}^{n} \circ e_{e(i)}\left[C_{i}\right. & \left.\sim \mathbf{B}^{n}(x, r)\right] \\
& \leqslant \mathcal{L}^{n}\left[e_{\varepsilon(i)}\left(C_{i}\right) \sim \mathbf{B}^{n}(x, r)\right]+\alpha(n-k) l \varepsilon(i)^{n-k+1},
\end{aligned}
$$


and estimate

$$
\begin{aligned}
\mathcal{L}^{n}\left[e_{e(i)}\left(C_{i}\right) \sim\right. & \left.\mathbf{B}^{n}(x, r)\right]+\mathcal{L}^{n}\left[e_{e(i)}\left(C_{i}\right) \cap \mathbf{B}^{n}(x, r)\right] \\
= & \mathcal{L}^{n} \circ e_{e(i)}(C)<\mathcal{L}^{n} \circ e_{e(i)}(C) \\
\leqslant & \mathcal{L}^{n} \circ e_{e(i)}\left[C_{i} \sim \mathbf{B}^{n}(x, r)\right] \\
& +\mathcal{L}^{n} \circ e_{e(i)} \circ e_{2 e(i)}\left[C_{i} \cap \mathbf{S}^{n-1}(x, r)\right]+\mathcal{L}^{n} \circ e_{\varepsilon(i)}(P) \\
\leqslant & \mathcal{L}^{n}\left[e_{\varepsilon(i)}\left(C_{i}\right) \sim \mathbf{B}^{n}(x, r)\right]+\alpha(n-k) l \varepsilon(i)^{n-k+1} \\
& +\alpha(n) 4^{n} \alpha(n-k) b c^{-1} l \varepsilon(i)^{n-k+1}+\gamma_{3} l^{k /(k-1)} \varepsilon(i)^{n-k} \\
\leqslant & \mathfrak{L}^{n}\left[e_{\varepsilon(i)}\left(C_{i}\right) \sim \mathbf{B}^{n}(x, r)\right]+\gamma_{4} l^{k /(k-1)} \varepsilon(i)^{n-k},
\end{aligned}
$$

for an appropriate $\gamma_{4} \in \mathbf{R}^{+}$. Conclusion (1) follows.

(2) Let $i, x$, and $r$ be as in (2). Define $f: \mathbf{R}^{+} \rightarrow \mathbf{R}$ by setting

$$
f(s)=\mathscr{g}_{i}\left[\mathbf{B}^{n}(x, s)\right],
$$

so $f$ is an increasing function with $f^{\prime}(s) \geqslant l_{i}(x, s)$ for $\mathcal{L}^{1}$ almost all positive $s$. For each $s$ with $\operatorname{dist}(x, B)>s \geqslant \varepsilon(i)$, we have

$$
f(s) \leqslant \Gamma_{1} f^{\prime}(s)^{k /(k-1)},
$$

thus

$$
\left[f(s)^{1 / k}\right]^{\prime} \geqslant k^{-1} \Gamma_{1}^{(1-k) / k}
$$

Conclusion (2) follows.

(3) Conclusion (3) follows from [FH, 3.2.22 and 3.2.39] and [RE, Lemma 2A].

(4) By (2) and (3) above we see that the $C_{i}$ are uniformly bounded. Then the existence of a subsequence as in (4) is a consequence of 3(2) and [FH, 2.5.2] together with the fact that $\mathcal{K}\left(\mathbf{R}^{n}\right)$ has a countable dense set. Conclusion (a) follows from 3(5). Conclusion (b) follows from (2). Conclusion (c) is clear. Conclusion (d) follows from (b), (c), (3), and [FH, 2.10.19(3)].

\section{Main theorems.}

7.1. Preliminaries. Throughout $\S 7$ we make the assumptions of 6.1. Further, we assume $B$ is $k-1$ rectifiable and that there exist $C \in \mathcal{C}$ and a Radon measure $g$ over $\mathbf{R}^{n}$ with compact support such that $\lim _{i} C_{i}=C$ in the Hausdorff distance topology and $g_{\text {is }}$ the weak limit of $g_{i}$ as $i \rightarrow \infty$.

7.2. Theorem. There exists $\Gamma_{2} \in \mathbf{R}^{+}$which does not depend on $B, L,\{\varepsilon(i)\}$, $\left\{C_{i}\right\}$, such that, for each $x \in C \sim B$ and $r \in \mathbf{R}^{+}$,

$$
\begin{aligned}
\mathcal{G}\left[\mathbf{B}^{n}(x, r)\right] \leqslant & \boldsymbol{\alpha}(k) r^{k} \\
& \cdot \sup \left\{\Gamma_{2}, \alpha(k)^{-1} k^{-1} \cdot \operatorname{diam}(B) \cdot \mathcal{F}^{k-1}(B) \cdot[\operatorname{dist}(x, B)]^{-k}\right\} .
\end{aligned}
$$


Proof. Let $r \in \mathbf{R}^{+}$and $i \in\{1,2,3, \ldots\}$ be such that $\mathbf{B}^{n}(x, r) \cap B=\varnothing$, $\mathbf{U}^{n}(x, r) \cap C_{i} \neq \varnothing$, and $r>\varepsilon(i)$. Proceed as in the proof of 6.2(1) to obtain the $k-1$ polyhedral complex $A$ with

$$
\mathscr{P}^{j}(A) \leqslant \boldsymbol{\alpha}(j)\left(3^{n} b\right)^{k-1} \boldsymbol{\alpha}(n-k) b c^{-1} \cdot \operatorname{l\varepsilon }(i)^{j+1-k}
$$

for each integer $j$, where $l=l_{i}(x, r)$. We will apply 5.5(3c) with $\theta=\tau r$ where $\tau<1$ has yet to be determined. By $5.5(3 \mathrm{c})$, provided $\varepsilon(i)<\theta$, we may choose $a \in \mathbf{A}$ so that

$$
\mathfrak{L}^{n} \circ e_{e(i)}[H(A, a, \theta)] \leqslant 2^{-n} \cdot 6(k+1) \gamma(n, k) \theta \sum_{j} \mathscr{P}^{j}(A) \varepsilon(i)^{n-j-1} .
$$

Set $H=H(A, a, \theta)$ and

$$
\begin{aligned}
D= & {\left[C_{i} \sim \mathbf{B}^{n}(x, r)\right] \cup e_{2 \varepsilon(i)}\left[C_{i} \cap \mathbf{S}^{n-1}(x, r)\right] \cup H } \\
& \cup\left[\cup\left\{\boldsymbol{\mu}_{\theta} \circ \tau_{a}\left(\mathbf{W}^{\prime}(z)\right): z \in \mathbf{Z}_{k}^{n}, \boldsymbol{\mu}_{\theta} \circ \tau_{a}\left(\mathbf{W}^{\prime}(z)\right) \subset \mathbf{B}^{n}(x, 2 n r)\right\}\right],
\end{aligned}
$$

so, by $8.4(1), \mathrm{D} \in \mathcal{C}(B, L)$. Hence one has

$$
\begin{aligned}
\mathcal{L}^{n}\left[e_{e(i)}\left(C_{i}\right) \sim\right. & \left.\mathbf{B}^{n}(x, r)\right]+\mathfrak{L}^{n}\left[e_{\varepsilon(i)}\left(C_{i}\right) \cap \mathbf{B}^{n}(x, r)\right] \\
< & \mathcal{L}^{n}\left[e_{e(i)}(D)\right] \\
\leqslant & \mathcal{L}^{n}\left[e_{e(i)}\left(C_{i}\right) \sim \mathbf{B}^{n}(x, r)\right]+\boldsymbol{\alpha}(n-k) l \varepsilon(i)^{n-k+1} \\
& +\alpha(n) 4^{n} \boldsymbol{\alpha}(n-k) b c^{-1} l \varepsilon(i)^{n-k+1} \\
& +2^{-n} \cdot 6(k+1) \gamma(n, k) \theta \sum_{j} \mathscr{P}^{j}(A) \varepsilon(i)^{n-j-1} \\
& +(12 n)^{n} \sum_{j=0}^{k} r^{j} \tau^{j-n} \boldsymbol{\alpha}(n-j) \varepsilon(i)^{n-j}
\end{aligned}
$$

provided $\tau \leqslant 1$ and $\varepsilon(i)<\theta$. Setting

$$
f(r)=\mathscr{g}_{i}\left[\mathbf{B}^{n}(x, r)\right]
$$

one has

$$
\beta f(r) \leqslant[\varepsilon(i)+\tau r] f^{\prime}(r)+\tau^{-n} r^{k}
$$

where $\beta<1$ depends only on $n$ and $k$. Set

$$
\tau=4^{-1} k^{-1} \beta \text { and } \Gamma_{2}=2 \tau^{-n} \beta^{-1} \alpha(k)^{-1} .
$$

Observe that, as a consequence of 6.2(3),

$$
\mathscr{g}_{i}\left[\mathbf{B}^{n}(x, r)\right] \leqslant \boldsymbol{\alpha}(k) r^{k}\left[\boldsymbol{\alpha}(k)^{-1} k^{-1} \cdot \operatorname{diam}(B) \cdot \mathcal{F}^{k-1}(B)[\operatorname{dist}(x, B)]^{-k}\right]
$$

whenever $r \geqslant \operatorname{dist}(x, B)$. If, for $r<\operatorname{dist}(x, B)$,

$$
f(r) \boldsymbol{\alpha}(k)^{-1} r^{-k} \geqslant \Gamma_{2},
$$


then one has

$$
f(r) \leqslant k^{-1} r f^{\prime}(r)
$$

provided $\varepsilon(i)<\tau r$. Thus if $(*)$ holds, then one sees that $f(r) \alpha(k)^{-1} r^{-k}$ is increasing. The conclusion of 7.2 follows.

7.3. THEOREM. The set $C$ is $\left(\mathcal{H}^{k}, k\right)$ rectifiable.

Proof. By 6.2(4d) and [FH 3.3.13] there exist Borel sets $S$ and $U$ such that $S \cap U=\varnothing, S \cup U=C, S$ is $\left(\mathcal{H}^{k}, k\right)$ rectifiable, $U$ is purely $\left(\mathcal{H}^{k}, k\right)$ unrectifiable, and $g_{1}^{k}(U)=0$. Set

$$
R=(U \sim B) \cap\left\{x: \Theta^{k}\left(\mathcal{H}^{k}\llcorner S, x)=0\right\}\right.
$$

and notice that by $[\mathbf{F H}, 2.10 .19(4)]$ it suffices to show $R=\varnothing$. By [FH, 2.10.15] we may assume $\mathcal{L}^{k}\left[\mathbf{p}_{\lambda}(U)\right]=0$ for each $\lambda \in \Lambda(n, k)$, where $\mathbf{p}_{\lambda}$ is as in [FH, 1.7.4].

Let $2^{-1}<\rho<1$ be arbitrary and set

$$
\sigma=3^{-(k+1)} n^{-k / 2}(1-\rho)^{k} \operatorname{card}[\Lambda(n, k)]^{-1} .
$$

Let $x \in R$ be arbitrary. There exists $r_{0} \in \mathbf{R}^{+}$, with $r_{0}<\operatorname{dist}(x, B)$, such that if $r<r_{0}$, then

$$
\mathcal{H}^{k}\left[\mathbf{B}^{n}(x, r) \cap S\right]<\sigma r^{k} .
$$

Let $r \leqslant r_{0}$ be arbitrary and set $s=\rho r, \theta=(r-s) 6^{-1} n^{-1 / 2}$.

For each $\lambda \in \Lambda(n, k)$ define $f_{\lambda}: \mathbf{R}^{n} \rightarrow\{0,1\}$ by setting $f_{\lambda}(a)=1$ if and only if

$$
\mathbf{p}_{\lambda}^{-1}\left[\mathbf{p}_{\lambda}(z)\right] \cap \boldsymbol{\tau}_{-a}^{\circ} \circ \boldsymbol{\mu}_{1 / \theta}\left[\mathbf{B}^{n}(x, r) \cap C\right] \neq \varnothing
$$

for some $z \in \mathbf{Z}_{n}^{n}$. Set

$$
f=\sum_{\lambda \in \Lambda(n, k)} f_{\lambda} \text { and } \mathbf{A}=\mathbf{R}^{n} \cap\{x: \mathbf{m}(x)<1\} .
$$

One estimates

$$
\begin{aligned}
\int_{\mathbf{A}} f(a) d \mathfrak{L}_{a}^{n} & =\sum_{\lambda \in \Lambda(n, k)} \int_{\mathbf{A}} f_{\lambda}(a) d \mathscr{L}_{a}^{n} \\
& <\operatorname{card}[\Lambda(n, k)] \sigma r^{k} \theta^{-k} 2^{n-k}=3^{-1} \mathfrak{L}^{n}(\mathbf{A}) .
\end{aligned}
$$

Hence there exists a compact $\mathbf{A}^{\prime} \subset \mathbf{A}$ with $\mathscr{L}^{n}\left(\mathbf{A}^{\prime}\right)=2 \cdot 3^{-1} \mathscr{L}^{n}(\mathbf{A})$ such that $f(a)=0$ for each $a \in \mathbf{A}^{\prime}$.

Choose an arbitrary $i \in\{1,2,3, \ldots\}$ which satisfies $\varepsilon(i)<\theta$. Proceed in a manner similar to the proof of 6.2(1) to construct a $k$ polyhedral complex $P$ from a covering of $C_{i} \cap\left[\mathrm{B}^{n}(x, r) \sim \mathrm{U}^{n}(x, s)\right]$ which satisfies

$$
\mathscr{P}^{j}(P) \leqslant \gamma_{1} \mathscr{g}_{i}\left[\mathbf{B}^{n}(x, r) \sim \mathbf{U}^{n}(x, s)\right] \varepsilon(i)^{j-k}
$$

for each integer $j$, where $\gamma_{1} \in \mathbf{R}^{+}$depends only on $n$ and $k$. 
For each $a \in \mathbf{A}$, set

$$
\begin{aligned}
& V^{\prime}(a)=\cup\left\{\operatorname{Clos} \mathbf{W}^{\prime}(z): z \in \mathbf{Z}_{n}^{n}\right. \text { with } \\
& \left.\boldsymbol{\tau}_{-a}{ }^{\circ} \boldsymbol{\mu}_{1 / \theta}\left[\mathbf{B}^{n}(x, s+\theta)\right] \cap \operatorname{Clos} \mathbf{W}^{\prime}(z) \neq \varnothing\right\}
\end{aligned}
$$

and

$$
V(a)=\mu_{\theta} \circ \tau_{a}\left[V^{\prime}(a)\right]
$$

Now, for $\mathfrak{L}^{n}$ almost all $a \in A, P \cap$ Bdry $V(a)$ can be made a $k-1$ polyhedral complex so as to satisfy

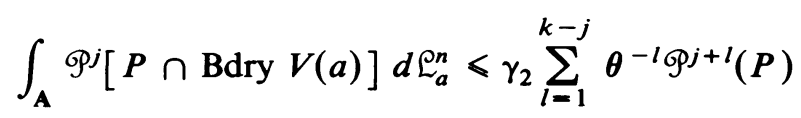

for $j=0,1,2, \ldots, k-1$, where $\gamma_{2} \in \mathbf{R}^{+}$depends only on $n$ and $k$. Thus we may select $b \in \mathbf{A}$ so that

$$
\mathscr{P}^{j}[P \cap \operatorname{Bdry} V(b)] \leqslant(k+1) \gamma_{2} \sum_{l=1}^{k-j} \theta^{-l} \mathcal{P}^{j+l}(P)
$$

for $j=0,1,2, \ldots, k-1$. By $5.5(3 \mathrm{c})$ one can choose $a_{i} \in \mathbf{A}^{\prime}$ so that

$$
\begin{aligned}
\mathcal{L}^{n} \circ e_{e(i)}[H(P \cap & \left.\left.\operatorname{Bdry} V(b), a_{i}, \theta\right)\right] \\
\leqslant & 3 \cdot 2^{-n-1} \cdot 6(k+1) \gamma(n, k) \theta \\
& \cdot \sum_{j} \mathcal{P}^{j}[P \cap \operatorname{Bdry} V(b)] \varepsilon(i)^{n-j-1} \\
\leqslant & \gamma_{3} \theta \sum_{j=0}^{k-1}(k+1) \gamma_{2} \sum_{l=1}^{k-j} \rho^{j+l}(P) \theta^{-l} \varepsilon(i)^{n-j-1} \\
\leqslant & (k+1) \gamma_{1} \gamma_{2} \gamma_{3} \cdot \theta g_{i}\left[\mathbf{B}^{n}(x, r) \sim \mathbf{U}^{n}(x, s)\right] \\
& \cdot \sum_{j=0}^{k-1} \sum_{l=1}^{k-j} \theta^{-l} \varepsilon(i)^{n-k+l-1} \\
\leqslant & \gamma_{4} g_{i}\left[\mathbf{B}^{n}(x, r) \sim \mathbf{U}^{n}(x, s)\right] \varepsilon(i)^{n-k},
\end{aligned}
$$

where $\gamma_{3}, \gamma_{4} \in \mathbf{R}^{+}$depend only on $n$ and $k$.

Set

$$
\begin{aligned}
C_{i}^{\prime}= & {\left[C_{i} \sim\left(\mathbf{B}^{n}(x, r) \sim \mathbf{U}^{n}(x, s)\right)\right] } \\
& \cup e_{2 \varepsilon(i)}\left[\left(C_{i} \cap \mathbf{S}^{n-1}(x, r)\right) \cup\left(C_{i} \cap \mathbf{S}^{n-1}(x, s)\right)\right] \cup P .
\end{aligned}
$$

By 8.4(2) we have $C_{i}^{\prime} \in \mathcal{C}(B, L)$. Now, $V(b) \subset \mathbf{B}^{n}(x, r)$ so if $\mu_{\theta} \circ \tau_{a_{i}} \circ \sigma_{k-1} \circ \tau_{-a_{i}} \circ \mu_{1 / \theta}\left(\sigma_{k-1}\right.$ as in [FH, 4.2.6]) is not defined on $C_{i}^{\prime} \cap$ $V(b)$, then there is $y_{i} \in C_{i}^{\prime} \cap \mathbf{B}^{n}(x, r)$ such that at least $k$ coordinates of $\tau_{-a_{i}} \circ \mu_{1 / \theta}\left(y_{i}\right)$ are even integers. Since $C_{i}$ converges to $C$ in the Hausdorff distance topology and $A^{\prime}$ is compact, it follows that 
$\mu_{\theta} \circ \tau_{a_{i}} \circ \sigma_{k-1} \circ \tau_{-a_{i}} \circ \mu_{1 / \theta}$ is defined on $C_{i}^{\prime} \cap V(b)$ for all sufficiently large $i$. Also, for sufficiently large $i$,

$$
C_{i}^{\prime} \cap \text { Bdry } V(b)=P \cap \text { Bdry } V(b)
$$

holds. It then follows that

$$
\begin{aligned}
& {\left[C_{i}^{\prime} \sim V(b)\right] } \cup H\left[P \cap \operatorname{Bdry} V(b), a_{i}, \theta\right] \\
& \cup \mu_{\theta} \circ \tau_{a_{i}} \circ \sigma_{k-1} \circ \tau_{-a_{i}} \circ \mu_{1 / \theta}\left[C_{i}^{\prime} \cap V(b)\right]
\end{aligned}
$$

is in $\mathcal{C}(B, L)$ for all sufficiently large $i$, and, hence, by [RE, Lemma 17A],

$$
\left[C_{i}^{\prime} \sim V(b)\right] \cup H\left[P \cap \operatorname{Bdry} V(b), a_{i}, \theta\right]
$$

is in $\mathcal{C}(B, L)$ for all sufficiently large $i$. So one has, for all sufficiently large $i$,

$$
\begin{aligned}
& \mathfrak{L}^{n}\left[e_{\varepsilon(i)}\left(C_{i}\right) \sim \mathbf{B}^{n}(x, r)\right]+\mathfrak{L}^{n}\left[e_{\varepsilon(i)}\left(C_{i}\right) \cap \mathbf{B}^{n}(x, r)\right] \\
& =\mathfrak{L}^{n} \circ e_{\mathrm{e}(i)}\left(C_{i}\right) \\
& \leqslant \mathscr{L}^{n}\left[e_{e(i)}\left(C_{i}\right) \sim \mathbf{B}^{n}(x, r)\right]+\mathscr{L}^{n} \circ e_{4 \mathrm{e}(i)}(P) \\
& +\mathfrak{L}^{n} \circ e_{e(i)}\left[H\left(P \cap \operatorname{Bdry} V(b), a_{i}, \theta\right)\right] \\
& \leqslant \mathcal{L}^{n}\left[e_{e(i)}\left(C_{i}\right) \sim \mathbf{B}^{n}(x, r)\right] \\
& +\gamma_{1} \mathscr{g}_{i}\left[\mathbf{B}^{n}(x, r) \sim \mathbf{U}^{n}(x, s)\right] \cdot \sum_{j=0}^{k} \alpha(n-j) 4^{n-j} \varepsilon(i)^{n-k} \\
& +\gamma_{4} \mathscr{g}_{i}\left[\mathbf{B}^{n}(x, r) \sim \mathbf{U}^{n}(x, s)\right] \varepsilon(i)^{n-k} .
\end{aligned}
$$

Thus one has

$$
\mathscr{G}\left[\mathbf{U}^{n}(x, r)\right] \leqslant \gamma_{5} \mathscr{g}\left[\mathbf{B}^{n}(x, r) \sim \mathbf{U}^{n}(x, s)\right]
$$

where $\gamma_{5} \in \mathbf{R}^{+}$depends only on $n$ and $k$. But $2^{-1}<\rho<1$ was arbitrary so, by $6.2(4 \mathrm{~b})$,

$$
\mathscr{g}\left[\mathbf{S}^{n-1}(x, r)\right] \geqslant \gamma_{5}^{-1} k^{-k} \Gamma_{1}^{1-k} r^{k},
$$

and this contradicts 7.2 .

7.4. Theorem. (1) There exists $\Gamma_{3} \in \mathbf{R}^{+}$such that for each $X \in \mathcal{C}$, each $\varepsilon \in \mathbf{R}^{+}$, and each Lipschitzian $f: X \rightarrow \mathbf{R}^{n}$ one has

$$
\mathfrak{L}^{n} \circ e_{\varepsilon}[f(X)] \leqslant \Gamma_{3}[1+\operatorname{Lip}(f)]^{n} \mathfrak{L}^{n} \circ e_{\varepsilon}(X) .
$$

(2) For each $\left(\mathcal{H}^{k}, k\right)$ rectifiable and $\mathcal{H}^{k}$ measurable set $X \subset \mathbf{R}^{n}$, if there exists $\beta \in \mathbf{R}^{+}$such that, for each $x \in X$ and each $r \in \mathbf{R}^{+}$,

$$
\mathbf{B}^{n}(x, r) \cap B=\varnothing \text { implies } \mathcal{F}^{k}\left[\mathbf{B}^{n}(x, r) \cap X\right]>\beta r^{k},
$$

then $\mathscr{T}^{k}(X)=\mathcal{H}^{k}(X)$.

(3) Let $X \in \mathcal{C}(B, L)$ satisfy

$$
\mathcal{F}^{k}(X)=\inf \left\{\mathcal{H}^{k}(Y): Y \in \mathcal{C}(B, L)\right\}
$$


and set $Z=\operatorname{spt}\left(\mathcal{H}^{k} L X\right) \cup B$; then $Z \in \mathcal{C}(B, L)$ and $\mathscr{T}^{k}(Z)=\mathcal{H}^{k}(Z)$.

(4) If $x \in C \sim B, f \in \mathbf{O}^{*}(n, k)$, and $g \in \mathbf{O}^{*}(n, n-k)$ are such that

$$
f^{*}\left(\mathbf{R}^{k}\right)=\operatorname{Tan}^{k}\left(\mathcal{H}^{k}\llcorner C, x) \text { and } g^{*}\left(\mathbf{R}^{n-k}\right)=\operatorname{ker}(f),\right.
$$

then there exist arbitrarily small $r \in \mathbf{R}^{+}$such that

$$
f\left(f^{-1}\left[\mathbf{U}^{k}(0, r)\right] \cap g^{-1}\left[\mathbf{U}^{n-k}(0, r)\right] \cap \boldsymbol{\tau}_{-x}\left(C_{i}\right)\right)=\mathbf{U}^{k}(0, r)
$$

for all sufficiently large integers $i$.

(5) One has
(a) $\lim _{i} \mathscr{g}_{i}\left(\mathbf{R}^{n}\right) \geqslant \mathcal{F}^{k}(C)$,
(b) $\mathcal{H}^{k}(C)=\mathfrak{T}^{k}(C)$,
(c) $\mathscr{T}^{\mathrm{k}}(C)=\inf \left\{\mathscr{T}_{*}^{k}(Y): Y \in \mathcal{C}(B, L)\right\}$,
(d) $\mathcal{H}^{k}(C)=\inf \left\{\mathcal{H}^{k}(Y): Y \in \mathcal{C}(B, L)\right\}$.

Proof. (1) Let $X, \varepsilon, f$ be as in (1). Set $l=\operatorname{Lip}(f)$. By [FH, 2.8.14] there exists a positive integer $b$, depending only on $n$, such that there are disjointed families, $F_{1}, F_{2}, F_{3}, \ldots, F_{b}$, of closed balls of radius $\varepsilon$ centered in $X$ with $X \subset \cup\left(\cup_{j} F_{j}\right)$. Now, any point of $f(X)$ is within $l \varepsilon$ of $f(c)$ for some $c \in\left\{\mathrm{Ct}(B): B \in \cup_{j} F_{j}\right\}$, so we have

$$
\varrho^{n} \circ e_{\varepsilon}[f(X)] \leqslant \operatorname{card}\left(\bigcup_{j} F_{j}\right) \cdot(1+l)^{n} \alpha(n) \varepsilon^{n} .
$$

For each $j=1,2,3, \ldots, b$,

$$
\operatorname{card}\left(F_{j}\right) \leqslant\left[\alpha(n) \varepsilon^{n}\right]^{-1} \mathcal{L}^{n} \circ e_{\varepsilon}(X)
$$

holds. Conclusion (1) follows with $\Gamma_{3}=b$.

(2) Let $X$ and $\beta$ be as in (2). Let $\sigma \in \mathbf{R}^{+}$be arbitrary. By [FH, 3.2.18] one can choose a compact, $k$ rectifiable $W \subset X$ with $\mathcal{H}^{k}(X \sim W)<\sigma^{k+1}$. For each $\varepsilon \in \mathbf{R}^{+}$set

$$
A_{\varepsilon}=X \sim\left[e_{\sigma \varepsilon}(W) \cup e_{\sigma \varepsilon}(B)\right] .
$$

By [FH, 2.8.14] there exists a positive integer $b$ such that, for each $\varepsilon \in \mathbf{R}^{+}$, there exist disjointed families, $F_{1}, F_{2}, F_{3}, \ldots, F_{b}$, of closed balls of radius $\sigma \varepsilon$ centered in $A_{\varepsilon}$ so that $A_{\varepsilon} \subset \cup\left(\cup_{j} F_{j}\right)$. The hypothesis of (2) implies $\operatorname{card}\left(F_{j}\right)$ $\leqslant \sigma \beta^{-1} \varepsilon^{-k}$ for $j=1,2,3, \ldots, b$, hence one has

$$
\mathcal{L}^{n} \circ e_{\varepsilon}\left(A_{\varepsilon}\right) \leqslant(1+\sigma)^{n} \alpha(n) b \sigma \beta^{-1} \varepsilon^{n-k} \text {. }
$$

Since

$$
\mathfrak{L}^{n} \circ e_{\varepsilon}(X) \leqslant \mathcal{L}^{n} \circ e_{(1+\sigma) \varepsilon}(B)+\mathfrak{L}^{n} \circ e_{(1+\sigma) \varepsilon}(W)+\mathfrak{L}^{n} \circ e_{\varepsilon}\left(A_{\varepsilon}\right),
$$

one concludes by [FH, 3.2.39] that

$$
\mathscr{T}^{* k}(X) \leqslant(1+\sigma)^{n-k} \mathcal{H}^{k}(W)+(1+\sigma)^{n} \alpha(n) b \sigma \beta^{-1} \alpha(n-k)^{-1} .
$$

Because $\sigma$ was arbitrary, conclusion (2) follows from [FH, 3.2.37].

(3) Let $X$ and $Z$ be as in (3). By [RE, Lemma 21A] there exists a minimal 
$Y \subset X$ with $Y \in \mathcal{C}(B, L)$. Let $x \in X \sim Z$ be arbitrary. By [FH, 2.10.25], there exists $r \in \mathbf{R}^{+}$such that $\mathbf{B}^{n}(x, r) \cap B=\varnothing$ and

$$
\mathcal{H}^{k-1}\left[X \cap \mathbf{S}^{n-1}(x, r)\right]=0,
$$

which implies

$$
H_{k-1}\left[Y \cap \mathbf{S}^{n-1}(x, r)\right]=0
$$

by [RE, Lemma 17A]. By 8.2(2), $Y \sim \mathbf{U}^{n}(x, r) \in \mathcal{C}(B, L)$, which implies $x \notin Y$. Since $x \in X \sim Z$ was arbitrary, one has $Y \subset Z$, so $Z \in \mathcal{C}(B, L)$ by [RE, Lemma 7A]. That $\mathfrak{T}^{k}(Z)=\mathcal{H}^{k}(Z)$ follows from (2) and the last paragraph on p. 37 of [RE].

(4) Without loss of generality assume $x=0$. For each $r \in \mathbf{R}^{+}$set

$$
V(r)=f^{-1}\left[\mathbf{B}^{k}(0, r)\right] \cap g^{-1}\left[\mathbf{B}^{n-k}(0, r)\right]
$$

and $U(r)=$ Int $V(r)$. Assume, contrary to (4), that there exists $r_{0} \in \mathbf{R}^{+}$, with $r_{0}<8^{-1} \operatorname{dist}(0, B)$, such that for each $r \in \mathbf{R}^{+}$with $r \leqslant r_{0}$ there exist infinitely many positive integers $i$ such that

$$
\mathbf{U}^{k}(0, r) \sim f\left[U(r) \cap C_{i}\right] \neq \varnothing .
$$

By 6.2(4b), 7.2, and [FH, 2.10.19(1) and (3)] there exist $\gamma_{1}, \gamma_{2}, \gamma_{3}$, and $\gamma_{4} \in \mathbf{R}^{+}$such that if

$$
y \in C \cap \mathbf{B}^{n}\left[0,2^{-1} \operatorname{dist}(0, B)\right]
$$

and $0<s<4^{-1} \operatorname{dist}(0, B)$, then

$$
\begin{aligned}
\gamma_{1} s^{k} & \leqslant \mathscr{G}\left[\mathbf{B}^{n}(y, s)\right] \leqslant \gamma_{2} \mathcal{F}^{k}\left[\mathbf{B}^{n}(y, s) \cap C\right] \\
& \leqslant \gamma_{3} \mathcal{g}\left[\mathbf{B}^{n}(y, s)\right] \leqslant \gamma_{4} s^{k} .
\end{aligned}
$$

Let $2^{-1}<\rho<1$ and $0<\xi<2^{-3 k / 2}(1-\rho)^{k} \gamma_{1} \gamma_{4}^{-1}$ be arbitrary. Choose $0<r_{1}<r_{0}$ such that

$$
\mathcal{H}^{k}\left(C \cap V(t) \sim g^{-1}\left[\mathbf{B}^{n-k}\left(0,2^{-1}(1-\rho) t\right)\right]\right) \leqslant \xi \mathcal{H}^{k}[C \cap V(t)]
$$

for each $t \leqslant r_{1}$. Let $0<r \leqslant r_{1}$ be arbitrary and notice that

$$
C \cap V\left[2^{-1}(1+\rho) r\right] \sim g^{-1}\left[\mathbf{B}^{n-k}(0,(1-\rho) r)\right]=\varnothing,
$$

since if $y$ is in the above set, then

$$
\mathbf{B}^{n}\left[y, 2^{-1}(1-\rho) r\right] \subset V(r) \sim g^{-1}\left[\mathbf{B}^{n-k}\left(0,2^{-1}(1-\rho) r\right)\right]
$$

and, consequently,

$$
\mathcal{H}^{k}\left(C \cap V(r) \sim g^{-1}\left[\mathbf{B}^{n-k}\left(0,2^{-1}(1-\rho) r\right)\right]\right) \geqslant 2^{-k}(1-\rho)^{k} \gamma_{1} r^{k} \gamma_{2}^{-1}
$$

while

$$
\mathcal{H}^{k}[V(r) \cap C] \leqslant \gamma_{4} 2^{k / 2} r^{k} \gamma_{2}^{-1}
$$

which is impossible by the choice of $\xi$ and $r$. 
By hypothesis, one can choose a subsequence $i(1), i(2), i(3), \ldots$ of 1,2 , $3, \ldots$ and for each $j=1,2,3, \ldots$ an $a_{j} \in \mathbf{U}^{k}(0, \rho r)$ such that $a_{j} \notin f[U(\rho r)$ $\left.\cap C_{i(j)}\right]$. For each $j=1,2,3, \ldots$ set $\bar{C}_{j}=C_{i(j)}, \bar{\varepsilon}(j)=\varepsilon(i(j))$, and $\bar{g}_{j}=g_{i(j)}$. Define a map $\Phi_{j}$ as follows:

(a) $\Phi_{j}$ is the identity on $\mathbf{R}^{n} \sim U\left[3^{-1}(1+2 \rho) r\right]$;

(b) for $x \in U\left[3^{-1}(1+2 \rho) r\right] \sim V\left[4^{-1}(1+3 \rho) r\right]$,

$$
\Phi_{j}(x)=t x+(1-t) f^{*} \circ f(x)
$$

where

$$
t=\left[|f(x)|-4^{-1}(1+3 \rho) r\right]\left[3^{-1}(1+2 \rho) r-4^{-1}(1+3 \rho) r\right]^{-1}
$$

(c) on $V\left[4^{-1}(1+3 \rho) r\right] \sim f^{-1}\left(f\left(a_{j}\right)\right) \Phi_{j}$ is $f^{*} \circ f$ followed by central projection from $a_{j}$ onto $f^{*}\left[\mathbf{S}^{k-1}\left(0,4^{-1}(1+3 \rho) r\right)\right]$.

We restrict our attention to $j$ such that

$$
d\left(\bar{C}_{j}, C\right)<6^{-1}(1-\rho) r \text { and } \bar{\varepsilon}(j)<4^{-1}(1-\rho) r .
$$

For such $j$, we have

$$
\bar{C}_{j} \cap V\left[3^{-1}(1+2 \rho) r\right] \sim g^{-1}\left[\mathbf{B}^{n-k}\left(0,6^{-1} \cdot 5(1-\rho) r\right)\right]=\varnothing,
$$

so $\Phi_{j}\left(\bar{C}_{j}\right) \in \mathcal{C}(B, L)$. Also, we have

$$
\begin{aligned}
e_{\bar{\varepsilon}(j)}\left[\Phi_{j}\left(\bar{C}_{j}\right)\right] \subset & {\left[e_{\bar{\varepsilon}(j)}\left(\bar{C}_{j}\right) \sim V(r)\right] } \\
& \cup\left[e_{\bar{\varepsilon}(j)}\left(\bar{C}_{j}\right) \cap V(r) \sim V(\rho r)\right] \\
& \cup e_{\bar{\varepsilon}(j)}\left[\Phi_{j}\left(\bar{C}_{j} \cap V\left(3^{-1}(1+2 \rho) r\right) \sim U\left(4^{-1}(1+3 \rho) r\right)\right)\right] \\
& \cup e_{\bar{\varepsilon}(j)}\left[f^{*}\left(\mathbf{S}^{k-1}\left(0,4^{-1}(1+3 \rho) r\right)\right)\right] .
\end{aligned}
$$

Now, one can check that

$$
\operatorname{Lip}\left[\Phi_{j} \mid \bar{C}_{j} \cap V\left(3^{-1}(1+2 \rho) r\right) \sim U\left(4^{-1}(1+3 \rho) r\right)\right] \leqslant 26
$$

holds. So, using (1), we obtain

$$
\begin{aligned}
\mathcal{L}^{n}\left[e_{\bar{\varepsilon}(j)}(\right. & \left.\left.\bar{C}_{j}\right) \cap V(r)\right] \leqslant \mathcal{L}^{n}\left[e_{\bar{\varepsilon}(j)}\left(\bar{C}_{j}\right) \cap V(r) \sim V(\rho r)\right] \\
& +\Gamma_{3}(27)^{n} \mathcal{L}^{n} \circ e_{\bar{\varepsilon}(j)}\left[\bar{C}_{j} \cap V\left(3^{-1}(1+2 \rho) r\right) \sim U\left(4^{-1}(1+3 \rho) r\right)\right] \\
& +\mathcal{L}^{n} \circ e_{\overline{\bar{\varepsilon}}(j)}\left[f^{*}\left(\mathbf{S}^{k-1}\left(0,4^{-1}(1+3 \rho) r\right)\right)\right] \\
\leqslant & {\left[1+\Gamma_{3}(27)^{n}\right] \mathcal{L}^{n}\left[e_{\bar{\varepsilon}(j)}\left(\bar{C}_{j}\right) \cap V(r) \sim V(\rho r)\right] } \\
& +2 k r^{k-1} \mathbf{\alpha}(k) \bar{\varepsilon}(j)^{n-k+1} .
\end{aligned}
$$

It follows that

$$
\mathscr{g}[V(r)] \leqslant\left[1+\Gamma_{3}(27)^{n}\right] \mathcal{g}[V(r) \sim V(\rho r)]
$$


holds for $0<r \leqslant r_{1}$ and from that we obtain

$$
g[V(r) \sim V(\rho r)] \geqslant \gamma_{1}\left[1+\Gamma_{3}(27)^{n}\right]^{-1} r^{k}
$$

for $0<r \leqslant r_{1}$. Thus we have

$$
\begin{aligned}
\mathcal{G}[V(r)] & =\sum_{j=0}^{\infty} \mathcal{g}\left[V\left(\rho^{j} r\right) \sim V\left(\rho^{j+1} r\right)\right] \\
& \geqslant \gamma_{1}\left[1+\Gamma_{3}(27)^{n}\right]^{-1} \cdot r^{k} \cdot \sum_{j=0}^{\infty} \rho^{j k} \\
& =\gamma_{1}\left[1+\Gamma_{3}(27)^{n}\right]^{-1}\left(1-\rho_{-}^{k}\right)^{-1} \cdot r^{k}
\end{aligned}
$$

for $0<r<r_{1}$. Since

$$
\mathcal{g}[V(r)] \leqslant \gamma_{3}^{-1} \gamma_{4} 2^{k / 2} r^{k}
$$

holds for $r \leqslant r_{0}$, we obtain a contradiction by choosing

$$
1>\rho>\left(1-\gamma_{1}\left[1+\Gamma_{3}(27)^{n}\right]^{-1} \gamma_{3} \gamma_{4}^{-1} 2^{-k / 2}\right)^{1 / k} .
$$

(5a) Conclusion (5a) follows from [FH, 2.8.7 and 3.2.19] and (4).

(5b) Let $\sigma \in \mathbf{R}^{+}$be arbitrary. By 6.2(4b), 6.2(4c), 7.2, and [FH, 2.10.19 $(1,3)], \mathcal{G}\left(\mathbf{R}^{n} \sim B\right)$ and $\mathcal{H}^{k} L(C \sim B)$ have the same sets of measure zero. Thus [FH, 3.2.18] allows us to choose a compact, $k$-rectifiable $W \subset C \sim B$ with $\mathscr{g}[C \sim(B \cup W)]<\sigma^{k+1}$. The proof now proceeds in a manner similar to the proof of (2), but we use $6.2(4 \mathrm{~b})$ to conclude

$$
\operatorname{card}\left(F_{j}\right) \leqslant \sigma k^{k} \Gamma_{1}^{k-1} \varepsilon^{-k} .
$$

(5c) From (5a), (5b) it follows that

$$
\Re^{k}(C)=\inf \left\{\Re^{* k}(Y): Y \in \mathcal{C}(B, L)\right\}
$$

holds. Let $D \in \mathcal{C}(B, L)$ be arbitrary and choose a new sequence $\{\varepsilon(1), \varepsilon(2)$, $\varepsilon(3), \ldots\} \subset \mathbf{R}^{+}$, with $\lim _{i} \varepsilon(i)=0$, so that

$$
\lim _{i} \alpha(n-k)^{-1} \varepsilon(i)^{k-n} \mathfrak{L}^{n} \circ e_{\varepsilon(i)}(D)=\mathfrak{N}_{*}^{k}(D) .
$$

Applying the previous results, one obtains $\bar{C} \in \mathcal{C}(B, L)$ such that, by (5a), $(5 b)$,

$$
\mathscr{T}^{k}(\bar{C})<\mathscr{T}^{k}(D)
$$

while, by $(\dagger)$ applied to $\bar{C}, \mathfrak{N}^{\mathrm{k}}(C)=\mathfrak{T}^{k}(\bar{C})$. This proves $(5 \mathrm{c})$.

(5d) Conclusion (5d) follows from (3), (5b), (5c), and the main theorem of [RE].

\section{Topological lemmas.}

8.1. Preliminaries. For use in 8.2 we fix the following terminology:

(1) $B, C, D, E, F \in \mathcal{C}$;

(2) $L \subset H_{k-1}(B)$ is a subgroup; 
(3) $\varepsilon \in \mathbf{R}^{+}$and $\mathcal{Q}$ is a finite covering of $D \cap C$ by open balls of radius no larger than $\varepsilon$;

(4) set $H=e_{e}[$ Clos $\cup \mathbb{Q}]$ and $A=\mathbf{N}(\mathbb{Q}, D \cap C)_{k-1}$;

(5) assume $E \in \mathcal{C}(B, L), F \cap B=\varnothing$, and $k \geqslant 2$;

(6) $i: D \cap C \rightarrow H, j: A \rightarrow H, l: B \rightarrow E \sim$ Int $F, m: E \cap$ Bdry $F \rightarrow E \sim$ Int $F$ are inclusion maps.

\subsection{Lemma. One has}

(1) $i_{*}\left[H_{k-1}(D \cap C)\right] \subset j_{*}\left[H_{k-1}(A)\right]$,

(2) $l_{*}(L) \subset m_{*}\left[H_{k-1}(E \cap\right.$ Bdry $\left.F)\right]$.

Proof. (1) Let $\mathscr{D}$ be an arbitrary covering of $D \cap C$ by open balls. Then $\mathscr{D}$ induces a covering of the space $D \cap C$, and the nerve, $\mathbf{N}_{\mathscr{D}}$, of this covering can be mapped to $\mathrm{N}(\mathscr{D}, D \cap C)$ in the obvious manner; we will denote this map by $f_{\oplus \text {. }}$.

Let $\sigma \in H_{k-1}(D \cap C)$ be arbitrary. Let $\mathscr{B}$ be an arbitrary covering of $D \cap C$ by open balls which refines $\mathscr{Q}$ and let $p: \mathbf{N}_{\mathscr{B}} \rightarrow \mathbf{N}_{\mathscr{Q}}$ be the projection (see [ES, IX, 2]). One notes that $r \circ f_{\mathscr{B}}$ is homotopic to $q \circ f_{\mathscr{Q}} \circ p$, where $r$ : $\mathbf{N}(\mathscr{B}, D \cap C) \rightarrow H$ and $q: \mathbf{N}(Q, D \cap C) \rightarrow H$ are the inclusion maps. Since $\mathscr{B}$ refines $\mathcal{Q}$ we have $p_{*} \circ \pi_{\mathscr{B}}=\pi_{\mathscr{Q}}$ and we see that

$$
r_{*} \circ f_{\mathscr{B} *} \circ \pi_{\mathscr{B}}(\sigma)=q_{*} \circ f_{\mathfrak{Q} *} \circ \pi_{\mathfrak{Q}}(\sigma) .
$$

By the continuity of Čech homology [ES, X, Theorem 2.1], one concludes

$$
i_{*}(\sigma) \in q_{*}\left[H_{k-1}(\mathrm{~N}(\mathscr{Q}, D \cap C))\right] \text {. }
$$

But, clearly, one has

$$
q_{*}\left[H_{k-1}(\mathbf{N}(\mathscr{Q}, D \cap C))\right] \subset j_{*}\left[H_{k-1}(A)\right] .
$$

(2) Consider the following commutative diagram in which all the homomorphisms are induced by inclusion maps.

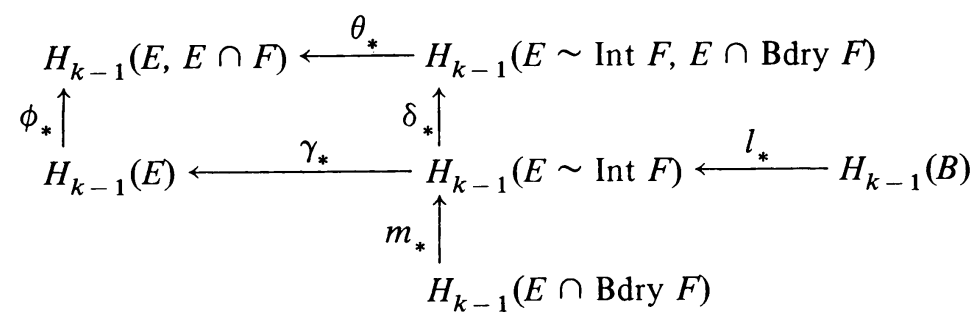

By [ES, IX, Theorem 7.6], $\operatorname{ker}\left(\delta_{*}\right)=\operatorname{im}\left(m_{*}\right)$, while, by [ES, X, Theorem 5.4], $\theta_{*}$ is an isomorphism. Let $\sigma \in L$ be arbitrary. By hypothesis $\gamma_{*}^{\circ} l_{*}(\sigma)=0$, so

$$
0=\phi_{*} \circ \gamma_{*} \circ l_{*}(\sigma)=\theta_{*} \circ \delta_{*} \circ l_{*}(\sigma) ;
$$

thus one has $\delta_{*} \circ l_{*}(\sigma)=0$, which implies $l_{*}(\sigma) \in \operatorname{im}\left(m_{*}\right)$. 
8.3. Preliminaries. For use in 8.4 we fix the following terminology:

(1) $B, C, D \in \mathcal{C}$;

(2) $L \subset H_{k-1}(B)$ is a subgroup;

(3) $\varepsilon \in \mathbf{R}^{+}, \mathbb{Q}$ is a finite covering of $D \cap C$ by open balls of radius no larger than $\varepsilon$, and $Q_{1} \subset Q$ is a covering of $\operatorname{Bdry}(D) \cap C$;

(4) set $A=\mathrm{N}(Q, D \cap C)_{k}$ and $A_{1}=\mathrm{N}\left(\mathbb{Q}_{1} \text {, Bdry }(D) \cap C\right)_{k-1}$;

(5) assume $C \in \mathcal{C}(B, L), D \cap B=\varnothing$, and $k \geqslant 2$.

8.4. TheOREM. (1) For each $Y \in \mathcal{C}\left[A_{1}, H_{k-1}\left(A_{1}\right)\right]$, one has

$$
(C \sim D) \cup e_{2 \varepsilon}[\operatorname{Bdry}(D) \cap C] \cup Y \in \mathcal{C}(B, L) .
$$

(2) One has

$$
(C \sim D) \cup e_{2 \varepsilon}[\operatorname{Bdry}(D) \cap(C)] \cup A \in \mathcal{C}(B, L) .
$$

Proof. (1) Set

$$
U=(C \sim D) \cup e_{2 \varepsilon}(C \cap \text { Bdry } D) \cup Y .
$$

Consider the following commutative diagram in which all the homomorphisms are induced by inclusion maps.

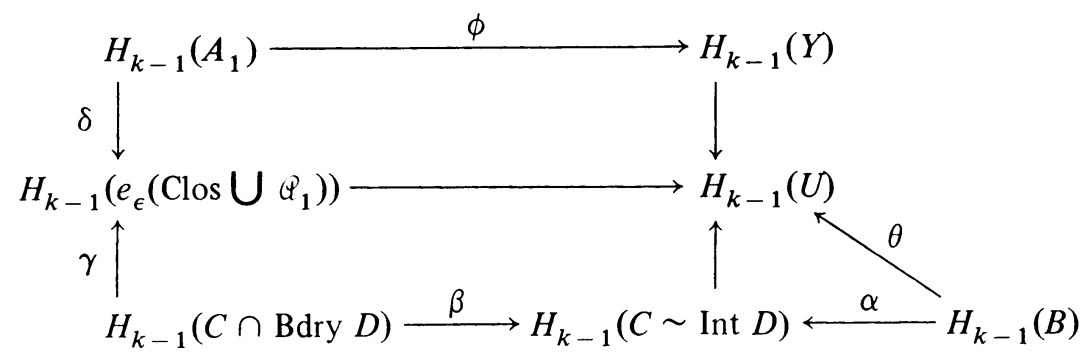

Let $\sigma \in L$ be arbitrary. By 8.2(2) there exists $\sigma^{\prime} \in H_{k-1}(\mathrm{C} \cap$ Bdry $D)$ such that $\beta\left(\sigma^{\prime}\right)=\alpha(\sigma)$. By 8.2(1) there exists $\sigma^{\prime \prime} \in H_{k-1}\left(A_{1}\right)$ such that $\delta\left(\sigma^{\prime \prime}\right)=$ $\gamma\left(\sigma^{\prime}\right)$. One sees easily that $\phi\left(\sigma^{\prime \prime}\right)=0$ implies $\theta(\sigma)=0$.

(2) Set

$$
\begin{gathered}
C_{1}=C \sim \text { Int } D, \quad C_{2}=C \cap D . \quad E=C \cap \text { Bdry } D, \\
V=(C \sim D) \cup e_{2 \varepsilon}[C \cap \text { Bdry } D] \cup A .
\end{gathered}
$$

Consider the following commutative diagram in which all homomorphisms, except $\partial_{1}, \partial_{2}$, and $\partial$ are induced by inclusion maps. 


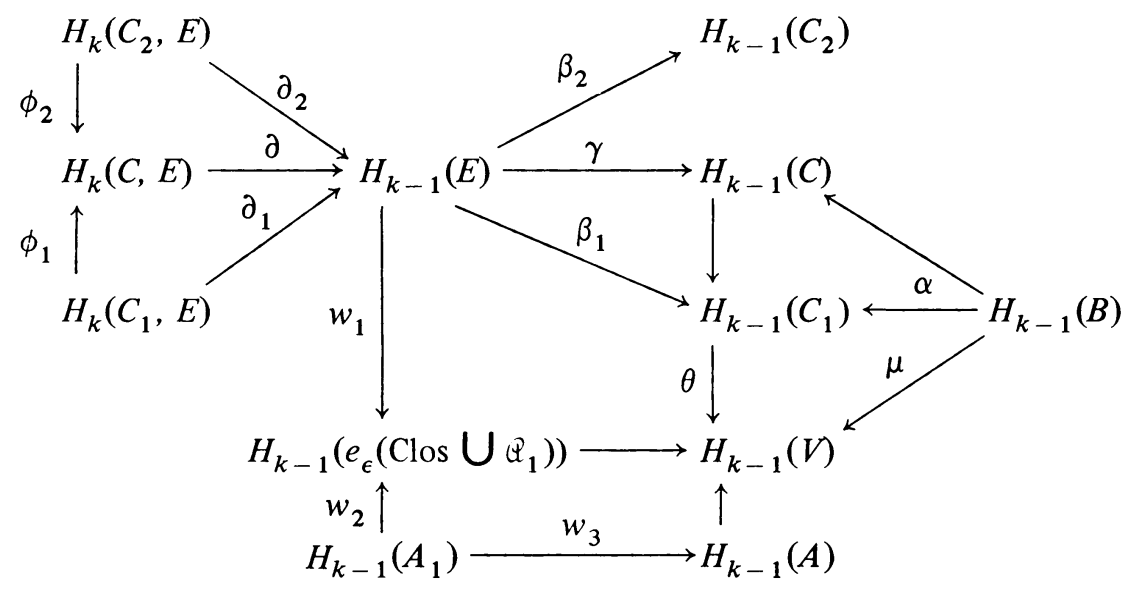

Let $\sigma \in L$ be arbitrary. By 8.2(2) there exists $\sigma^{\prime} \in H_{k-1}(E)$ such that $\beta_{1}\left(\sigma^{\prime}\right)=\alpha(\sigma)$. Since $C \in \mathcal{C}(B, L)$, one has $\gamma\left(\sigma^{\prime}\right)=0$. Thus there exists $\tau^{\prime} \in$ $H_{k}(C, E)$ such that $\partial\left(\tau^{\prime}\right)=\sigma^{\prime}$. The maps $\phi_{1}$ and $\phi_{2}$ provide an injective representation of $H_{k}(C, E)$ as a direct sum, so there exists $\tau_{i}^{\prime} \in H_{k}\left(C_{i}, E\right)$, for $i=1$, 2, such that $\tau^{\prime}=\phi_{1}\left(\tau_{1}^{\prime}\right)+\phi_{2}\left(\tau_{2}^{\prime}\right)$. Set $\sigma_{i}^{\prime}=\partial_{i}\left(\tau_{i}^{\prime}\right)$ for $i=1$, 2. One has $\sigma^{\prime}=\sigma_{1}^{\prime}+\sigma_{2}^{\prime}$ and $\beta_{1}\left(\sigma_{1}^{\prime}\right)=0$. Now by $8.2(1)$ there exists $\sigma_{2}^{\prime \prime} \in H_{k-1}\left(A_{1}\right)$ so that $w_{2}\left(\sigma_{2}^{\prime \prime}\right)=w_{1}\left(\sigma_{2}^{\prime}\right)$, and since $\beta_{2}\left(\sigma_{2}^{\prime}\right)=0$, one sees by the proof of $8.2(1)$ that $w_{3}\left(\sigma_{2}^{\prime \prime}\right)=0$. It follows that $\theta \circ \beta_{1}\left(\sigma_{2}^{\prime}\right)=0$, hence that $\theta \circ \beta_{1}\left(\sigma^{\prime}\right)=0$, and finally that $0=\theta \circ \alpha(\sigma)=\mu(\sigma)$.

Acknowledgement. My thanks to Professor Frederick J. Almgren, Jr., whose assistance and encouragement were indispensable. Thanks are also due the referee for his helpful comments.

The research for this paper was done while the author was supported by a National Science Foundation Graduate Fellowship.

\section{REFERENCES}

[BR] R. Bellman, Introduction to matrix analysis, 2nd ed., McGraw-Hill, New York, 1970.

[EH] S. Eilenberg and O. G. Harrold, Jr., Continua of finite linear measure. I, Amer. J. Math. 65 (1943), 137-146.

[ES] S. Eilenberg and N. Steenrod, Foundations of algebraic topology, Princeton Univ. Press, Princeton, N.J., 1952.

[FH] H. Federer, Geometric measure theory, Springer-Verlag, New York, 1969.

[RE] E. R. Reifenberg, Solution of the Plateau problem for m-dimensional surfaces of varying topological type, Acta Math. 104 (1960), 1-92.

Department of Mathematics, Oregon State University, Corvallis, Oregon 97331 